Pacific Journal of Mathematics

TOPOLOGICAL BOOLEAN RINGS. DECOMPOSITION OF 


\title{
TOPOLOGICAL BOOLEAN RINGS. DECOMPOSITION OF FINITELY ADDITIVE SET FUNCTIONS
}

\author{
HANS WEBER
}

\begin{abstract}
As a basis for the whole paper we establish an isomorphism between the lattice $\mathfrak{M}_{s}(R)$ of all $s$-bounded monotone ring topologies on a Boolean ring $R$ and a suitable uniform completion of $R$; it follows that $\mathfrak{M}_{s}(R)$ itself is a complete Boolean algebra. Using these facts we study $s$-bounded monotone ring topologies and topological Boolean rings (conditions for completeness and metriziability, decompositions). In the second part of this paper we give a simple proof of a Lebesgue-type decomposition for finitely additive (e.g. semigroup-valued) set functions on a ring, which was first proved by Traynor (in the group-valued case) answering a question of Drewnowski. Using the Lebesgue-decomposition various other decompositions are obtained.
\end{abstract}

0. Introduction. The first part of this paper (Chapter 2) deals with an examination of the lattice $\mathfrak{M}_{s}(R)$ of all $s$-bounded monotone ring topologies ( $=F N$-topologies) on a Boolean ring $R$. In (2.2) an isomorphism is established between $\mathfrak{M}_{s}(R)$ and the completion of $R$ for the finest $s$-bounded monotone ring topology $\mathbf{U}_{s}$ on $R$. From this result we get some consequences (criteria for completeness and metrizability, decomposition theorems for monotone ring topologies) which are also interesting for measure theory and which - as far as they are known in special cases - were before in each case proved with quite different methods.

In the second part of this paper (Chapter 3) a decomposition $\mu=$ $\Sigma_{\alpha \in A} \mu_{\alpha}$ into an infinite sum is given for an s-bounded content $\mu: R \rightarrow G$ defined on a Boolean ring with values in e.g. a complete Hausdorff topological group (content = finitely additive set function); this decomposition includes the usual decomposition theorems as special cases. For an illustration of the method Chapter 3 first deals with the case $|A|=2$, i.e. with the Lebesgue decomposition; this again includes as special cases decompositions of a Hewitt-Yosida type, decompositions into an atomless and an atomic content, into a regular and an antiregular content and others. We explain the arising problems with the Lebesgue decomposition. The classic Lebesgue decomposition $(\mu=\lambda+\nu, \lambda \perp u, \nu \ll u)$ of a nonnegative ( $\sigma$-additive) measure on a $\sigma$-algebra rests on a decomposition of the basic set into two disjoint sets of the $\sigma$-algebra. The same is still true 
for the Lebesgue decomposition of group-valued measures on a $\sigma$-complete Boolean ring satisfying the countable chain condition (CCC) [13, Corollary 2]. But things are different if measures without $\mathrm{CCC}$ or the finitely additive case are considered. In [20] Traynor - essentially generalizing a method of Rickart [18] - has decomposed $s$-bounded group-valued contents on rings with ideals which do not decompose the ring. Drewnowski asked in [5] if a stronger version of a Lebesgue decomposition (with a more adequate notion of singularity) is true. In [21] Traynor obtained such a more general and final version of a Lebesgue decomposition theorem which cannot be obtained from [20, Theorem 1.7] (s. Chapter $3)$. In the present paper this theorem of Traynor is also a simple corollary of the already mentioned Theorem (2.2). For that first the content $\mu$ is continuously extended to a content $\tilde{\mu}$ on the completion $\tilde{R}$ of $\left(R, \mathbf{U}_{s}\right)$. The decomposition $\tilde{\mu}=\tilde{\lambda}+\tilde{\nu}$ of $\tilde{\mu}$ yields the decomposition $\mu=\lambda+\nu$ by restriction $\lambda=\tilde{\lambda}|R, \nu=\tilde{\nu}| R$. The decomposition of $\tilde{\mu}$ is as in the classic case based on a decomposition of the Boolean algebra $\tilde{R}$. It is worth mentioning that $\tilde{R}$ is (as a lattice) a complete Boolean algebra and $\tilde{\mu}$ $\tau$-smooth (and so a measure). Considering that the examination of $\tilde{\mu}$ instead of $\mu$ is in combination with (2.2) comparable to the Stone space technique as it is used e.g. in [4], [6, Chapter 4], [11], [21, 6.2], [22]. Are contents with range in a non-metrizable space examined, the method used here and partly already in [24], [25] is often more suitable. That is due to the fact that the monotone ring topology induced by the "extension" $\overline{\bar{\mu}}$ of $\mu$ which the Stone space technique yields (notion as in $[21,6.2]$ ) is in general not complete in case of non-metrizable range space.

1. Preliminaries, notions. Throughout the paper let $R$ be a Boolean ring.

We as usual denote the symmetric difference (addition, subtraction), infimum (multiplication), supremum, difference, natural ordering by $\Delta$, $\wedge, \vee, \backslash, \leq$, respectively, and, if $R$ is a Boolean algebra, the unit by $e$. A subset $M$ of $R$ is called normal (in $R$ ) if $\{a \wedge b: a \in R, b \in M\}=$ : $R \wedge M \subset M \neq \varnothing$. A topology on $R$ with continuous subtraction and multiplication and a 0 -neighbourhood base of normal sets, is called a monotone ring topology; we always identify a monotone ring topology with the 0-neighbourhood system $\mathbf{U}$ belonging to it; $N(\mathbf{U}):=\bigcap_{U \in \mathbf{U}} U$ is the closure of $\{0\}$ with respect to $U$. The set $\mathfrak{M}(R)$ of all monotone ring topologies of $R$ forms a complete lattice with the trivial topology as its minimal element and the discrete topology as its maximal element. For $\mathbf{U} \in \mathfrak{M}(R)$ put $V(\mathbf{U}):=\{\mathbf{V} \in \mathfrak{M}(R): \mathbf{V} \subset \mathbf{U}\}$. We call $\mathbf{U}, \mathbf{V} \in \mathfrak{M}(R)$ 
singular if $\inf \{\mathbf{U}, \mathbf{V}\}$ is the trivial topology or equivalent to that $U \vee V=R$ for all $U \in \mathbf{U}$ and $V \in \mathbf{V}$ as you can see from the first of the following statements.

(1.1) For $\mathbf{U}, \mathbf{V} \in \mathfrak{M}(R)$,

$$
\{U \vee V: U \in \mathbf{U}, V \in \mathbf{V}\} \quad \text { is a base of } \mathbf{U} \wedge \mathbf{V}:=\inf \{\mathbf{U}, \mathbf{V}\}
$$

and

$$
\{U \wedge V: U \in \mathbf{U}, V \in \mathbf{V}\} \quad \text { is a base of } \mathbf{U} \vee \mathbf{V}:=\sup \{\mathbf{U}, \mathbf{V}\} .
$$

It follows from (1.1) that $\mathfrak{M}(R)$ is a distributive lattice. For an arbitrary cardinal number $\kappa$ we call $\mathbf{U} \in \mathfrak{M}(R) \kappa$-smooth if every decreasingly directed subset $M$ of $R$ with power $|M| \leq \kappa$ and inf $M=0$ converges to 0 with respect to $U$; for countable $\kappa$ and $\kappa=|R|$ we also say $\sigma$-smooth and $\tau$-smooth, respectively. An ideal $A$ of $R$ is said to be $\kappa$-complete provided for any $M \subset A$ with $|M| \leq \kappa$ the supremum of $M$ exists and belongs to $A$.

(1.2) Let $\kappa$ be an infinite cardinal number, $R$-complete and $\mathbf{U} \in \mathfrak{M}(R)$ $\kappa$-smooth.

Then $N(\mathbf{U})$ and $R / N(\mathbf{U})$ are $\kappa$-complete and the quotient topology on $(R, \mathbf{U}) / N(\mathbf{U})$ is $\kappa$-smooth.

Proof. The $\kappa$-completeness of $N(\mathrm{U})$ is obvious; hence, by [19, 21.1], $R / N(\mathbf{U})$ is $\kappa$-complete. For the proof of the $\kappa$-smoothness of the quotient topology $\hat{\mathbf{U}}$ of $R / N(\mathbf{U})$ let $\varphi: R \rightarrow R / N(\mathbf{U})$ be the canonical mapping, $M \subset R,|M| \leq \kappa, \varphi(M)$ decreasingly directed and $\inf M=0$. Then $M_{0}:=\{\inf E: E \subset M,|E|<\infty\}$ is decreasingly directed and $\left|M_{0}\right| \leq \kappa$; so $M_{0}$ converges to $a:=\inf M_{0}$ with respect to $\mathrm{U}$ and therefore $\varphi\left(M_{0}\right)$ to $\varphi(a)$ with respect to $\hat{\mathbf{U}}$. Hence, $\varphi(a)=\inf \varphi\left(M_{0}\right)$ (s. [24, (1.1)]), and, since $\varphi(M)$ is a cofinal subset of $\varphi\left(M_{0}\right), \varphi(a)=0$ is the limit of $\varphi(M)$ with respect to $\hat{\mathbf{U}}$.

$\mathbf{U} \in \mathfrak{M}(R)$ is called $s$-bounded if every disjoint sequence in $R$ converges to 0 with respect to $U$. The supremum $\mathbf{U}_{s}$ of all $s$-bounded monotone ring topologies on $R$ is also $s$-bounded and therefore the finest $s$-bounded monotone ring topology on $R$; so $\mathfrak{M}_{s}(R):=\{\mathbf{U} \in \mathfrak{M}(R): \mathbf{U}$ is $s$-bounded $\}=V\left(\mathbf{U}_{s}\right)$. As noted in the proof of $[24,(6.8)] \mathbf{U}_{s}$ is Hausdorff. The following theorem of [25] is essential for the whole paper.

(1.3) THEOREM (s. [25; (1.7), (2.1.3)]). (a) If $\mathbf{U}$ is a complete, s-bounded, Hausdorff monotone ring topology on $R$, then $(R, \leq)$ is (as lattice) complete and $\mathbf{U}$ is $\tau$-smooth. 
(b) If $(R, \leq)$ is complete and $\mathbf{U}$ and $\mathbf{V}$ are $\tau$-smooth monotone ring topologies on $R$ with $N(\mathbf{U}) \subset N(\mathbf{V})$, then $\mathbf{U} \supset \mathbf{V}$.

For a comparison with other papers especially with Traynor's we have to point out the connection between the different singularity conditions. Here and for the whole paper we use the following notion: For $a \in R$ and $\mathbf{U} \in \mathfrak{M}(R), p_{\mathbf{U}}(a):=\{(U \wedge a) \vee(R \backslash a): U \in \mathbf{U}\}$ denotes the monotone ring topology on $R$ such that $x \mapsto(x \wedge a, x \backslash a)$ is a topological isomorphism from $\left(R, p_{\mathbf{U}}(a)\right)$ onto $(R \wedge a, \mathbf{U} \mid R \wedge a) \times\left(R \backslash a, \mathbf{U}_{t}\right)$; here $\mathbf{U}_{t}$ stands for the trivial topology on $R \backslash a ; p_{\mathrm{U}}(a)$ is uniquely determined by the equalities $p_{\mathbf{U}}(a)|R \wedge a=\mathbf{U}| R \wedge a$ and $p_{\mathbf{U}}(a) \mid R \backslash a=\mathbf{U}_{t}$.

(1.4) For $\mathbf{U}, \mathbf{V} \in \mathfrak{M}(R)(\mathrm{S} 1),(\mathrm{S} 2)$, (S3) denote the following singularity conditions:

(S1) For all $U \in \mathbf{U}$ and $V \in \mathbf{V}$ there exists an element $a \in V$ with $\{x \in R: x \wedge a=0\} \subset U$;

(S2) $\mathbf{U}, \mathbf{V}$ are singular;

(S3) $\left\{a \in R: p_{\mathbf{U}}(a) \subset \mathbf{V}\right\}=N(\mathbf{U})$.

Then we have: (a) $(\mathrm{S} 1) \cap(\mathrm{S} 2) \cap(\mathrm{S} 3)$.

(b) If $U$ is $s$-bounded or $R$ has a unit, then (S1) and (S2) are equivalent.

(c) There are $s$-bounded monotone ring topologies $\mathbf{U}, \mathbf{V}$ on some Boolean algebra satisfying (S3), but not (S2).

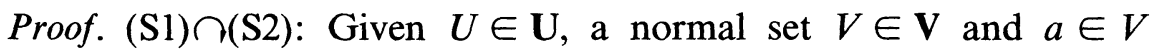
chosen as in (S1). Then we have $x=(x \backslash a) \vee(x \wedge a) \in U \vee V$ for all

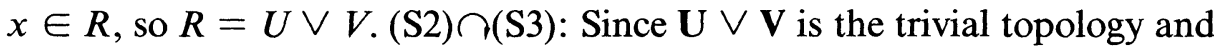
$p_{\mathbf{U}}(a) \subset \mathbf{U}$ for all $a \in R$ the following statements are equivalent: $p_{\mathbf{U}}(a)$ $\subset \mathbf{V}, p_{\mathbf{U}}(a) \subset \mathbf{U} \wedge \mathbf{V}, p_{\mathbf{U}}(a)$ is trivial, $a \in N(\mathbf{U})$.

(b) For the proof of $(\mathrm{S} 2) \cap(\mathrm{S} 1)$ we only need that the completion of $(R, \mathbf{U}) / N(\mathbf{U})$ has a unit. In each case, given $U \in \mathbf{U}, V \in \mathbf{V}$ and a normal set $U_{0} \in \mathbf{U}$ with $U_{0} \vee U_{0} \subset U$ there exists an element $c \in R$ with $\{x \in R$ : $x \wedge c=0\} \subset U_{0}$. By (S2) there are elements $a \in V$ and $b \in U_{0}$ with $a \vee b=c$. So for all $x \in R$ with $x \wedge a=0$ we have $x=(x \backslash c) \vee(x \wedge b)$ $\in U_{0} \vee U_{0} \subset U$.

(c) For an arbitrary nonnegative content $\mu: R \rightarrow[0, \infty[$ we denote by $\mathbf{W}_{0}(\mu)$ the monotone ring topology with $\{x \in R: \mu(x)<\varepsilon\}, \varepsilon>0$, as base of 0 -neighbourhoods. Now let $R$ be the Boolean algebra generated by all finite subsets of the set $\mathbf{N}$ of natural numbers and nonnegative contents $\mu_{1}, \mu_{2}$ on $R$ defined by $\mu_{1}(\mathbf{N})=\mu_{2}(\mathbf{N})=1, \mu_{1}(\{n\})=0, \mu_{2}(\{n\})=2^{-n}$ 
( $n \in \mathbf{N})$; put $\mu=\mu_{1}+\mu_{2}$. Then $\mathbf{U}:=\mathbf{W}_{0}(\mu)$ and $\mathbf{V}:=\mathbf{W}_{0}\left(\mu_{1}\right)$ have the desired properties.

The singularity conditions (S1), (S2), (S3) correspond in [21] to the notions singular, extremely noncontinuous, nowhere continuous, respectively. The example above shows that the statement (3) of [21, Theorem 3.3] is not true; in [20, Theorem 2.4] the uniqueness statement isn't valid from which with [21, Theorem 1.2] the statement (3) of [21, Theorem 3.3] would follows. ( $\mu=0+\mu=\mu_{1}+\mu_{2}$ yield different decompositions of $\mu$ with respect to $\mu_{1}$ in the sense of [20, Theorem 2.4], $\mu, \mu_{1}, \mu_{2}$ as above.)

In [23] we introduced the notion of independent topologies which is important in connection with certain questions of topoloical algebra. It is interesting that two monotone ring topologies on $R$ are singular iff they are independent.

2. The lattice of all $s$-bounded monotone ring topologies. The key to the study of $\mathfrak{M}_{s}(R)$ is the following lemma and theorem (2.2), part (a) of which allows one to reduce some examinations to the complete case.

(2.1) Key Lemma. Assume $\mathbf{U}$ is a Hausdorff monotone ring topology on $R$.

(a) $v_{1} \leq v_{2}$ iff $p_{\mathbf{U}}\left(v_{1}\right) \subset p_{\mathbf{U}}\left(v_{2}\right)$ for all $v_{1}, v_{2} \in R$.

(b) If $R$ is a Boolean algebra and $v \in R, \mathbf{V}=p_{\mathbf{U}}(v)$, then $N(\mathbf{V})=R \backslash v$, $v=e \backslash \sup N(\mathbf{V})$ and $x \mapsto x \Delta N(\mathbf{V})$ is a topological isomorphism from $(R \wedge$ $v, \mathbf{U} \mid R \wedge v)$ onto $(R, \mathbf{V}) / N(\mathbf{V})$.

(c) If $(R, \leq)$ is complete (as lattice) and $\mathbf{U} \tau$-smooth, then $p_{\mathbf{U}}$ : $v \mapsto p_{\mathbf{U}}(v)$ is a lattice isomorphism from $R$ onto $V(\mathbf{U})$.

Proof. (a), (b) are simple consequences of the definition of $p_{\mathrm{U}}(v)$. For (c) we have, because of (a), only to show that $p_{U}(a)$ is surjective. Let be $\mathbf{V} \in V(\mathbf{U})$. Since with $\mathbf{U}$ also $\mathbf{V}$ is $\tau$-smooth, $s:=\sup N(\mathbf{V}) \in N(\mathbf{U})$ (s. (1.2)). Put $v:=e \backslash s$, then $N\left(p_{\mathrm{U}}(v)\right)=R \backslash v=N(\mathbf{V})$, hence by (1.3)(b) $p_{\mathbf{U}}(v)=\mathbf{V}$. For a Hausdorff topology $\mathbf{U} \in \mathfrak{M}(R)$ the completion $(\tilde{R}, \tilde{\mathbf{U}})$ of $(R, \mathbf{U})$ is a Boolean ring and its topology $\tilde{\mathbf{U}}$ is a monotone ring topology.

(2.2) THEOREM. If $\mathbf{U}$ is a Hausdorff topology from $\mathfrak{M}_{s}(R)$ and $(\tilde{R}, \tilde{\mathbf{U}})$ is the completion of $(R, \mathbf{U})$, then we have:

(a) $\tilde{\mathbf{U}} \in \mathfrak{M}_{s}(\tilde{R}) ; \rho: \mathbf{V} \mapsto \tilde{\mathbf{V}} \mid R$ is a lattice isomorphism from $V(\tilde{\mathbf{U}})$ onto $V(\mathbf{U})$.

(b) $(\tilde{R}, \leq)$ is a complete Boolean algebra and $V(\tilde{\mathbf{U}})$ exactly consists of all $\tau$-smooth monotone ring topologies on $\tilde{R}$. 
(c) $V(\mathbf{U})$ is a complete Boolean algebra and $\pi: v \mapsto p_{\tilde{\mathbf{U}}}(v) \mid R$ a lattice and ring isomorphism from $\tilde{R}$ onto $V(\mathbf{U})$.

(d) If $v \in \tilde{R}$ and $\mathbf{V}=\pi(v), x \triangle N(\mathbf{V}) \mapsto x \wedge v$ is a topological isomorphism from $(R, \mathbf{V}) / N(\mathbf{V})$ onto a dense subring of $(\tilde{R} \wedge v, \tilde{\mathbf{U}} \mid \tilde{R} \wedge v)$ (and in this sense $(\tilde{R} \wedge v, \tilde{\mathbf{U}} \mid \tilde{R} \wedge v)$ is the completion of $(R, \mathbf{V}) / N(\mathbf{V})$.)

Proof. (a) is valid by the bit more general result [24, (1.8)].

(b) Since $\tilde{\mathbf{U}}$ is $s$-bounded (s. (a)), by (1.3)(a) $(\tilde{R}, \leq)$ is complete and $\tilde{\mathbf{U}}$ $\tau$-smooth, so every $\tilde{\mathbf{V}} \in V(\tilde{\mathbf{U}})$ is $\tau$-smooth. Vice versa every $\tau$-smooth monotone ring topology on $\tilde{R}$ belongs to $V(\tilde{\mathbf{U}})$ by $(1.3)(\mathrm{b})$.

(c) By (a) $\rho$ is a lattice isomorphism, by (b) and (2.1)(c) $p_{\tilde{\mathbf{U}}}$ is one; so $\pi=\rho \circ p_{\tilde{\mathbf{U}}}$ is also a lattice isomorphism. From this all statements of (c) follow. (d) follows from $(2.1)(\mathrm{b})$ and the fact that $\tilde{\mathbf{V}}=p_{\tilde{\mathbf{U}}}(v), x \triangle$ $N(\mathbf{V}) \mapsto x \triangle N(\tilde{\mathbf{V}})$ is a topological isomorphism from $(R, \mathbf{V}) / N(\mathbf{V})$ onto a dense subring of $(\tilde{R}, \tilde{\mathbf{V}}) / N(\tilde{\mathbf{V}})$.

(2.3) COROllaRY. Let be $\mathbf{U}, \mathbf{V} \in \mathfrak{M}_{s}(R)$ with $\mathbf{V} \subset \mathbf{U}$ :

(a) If $\mathbf{U}$ is complete, then $\mathbf{V}$ is complete, too.

(b) If for a cardinal number $\kappa \mathbf{U}$ possesses a 0 -neighbourhood base of power $\leq \kappa$, then $\mathbf{V}$ possesses a 0-neighbourhood base of power $\leq \kappa$, too.

Proof. We may assume that $\mathbf{U}$ is Hausdorff, otherwise consider $(R, \mathbf{U}) / N(\mathbf{U})$ endowed with the corresponding quotient topologies. With the notation of (2.2) put $v:=\pi^{-1}(\mathbf{V})$. With $\mathbf{U}$ also $\tilde{\mathbf{U}} \mid R \wedge v$ has a 0 -neighbourhood base of power $\leq \kappa$; since $(\tilde{R} \wedge v, \tilde{\mathbf{U}} \mid \tilde{R} \wedge v)$ is by $(2.2)(\mathrm{d})$ the completion of $(R, \mathbf{V}) / N(\mathbf{V})$, we get (b) from this. Is $\mathbf{U}$ complete, then $\mathbf{V}=p_{\mathbf{U}}(v)$ is obviously also complete.

(2.4) Corollary. For complete $\mathbf{U} \in \mathfrak{M}_{s}(R)$ two topologies $\mathbf{V}_{1}, \mathbf{V}_{2} \in$ $V(\mathbf{U})$ are singular iff $N\left(\mathbf{V}_{1}\right) \vee N\left(\mathbf{V}_{2}\right)=R$.

For the proof we may assume that $\mathbf{U}$ is Hausdorff. With $v_{i}:=p_{\mathbf{U}}^{-1}\left(\mathbf{V}_{l}\right)$ $(i=1,2), N\left(\mathbf{V}_{1}\right) \vee N\left(\mathbf{V}_{2}\right)=R$ by $(2.1)(\mathrm{b})$ just means that $v_{1} \wedge v_{2}=0$. Now observe that with the isomorphism in $(2.2)(\mathrm{c})$ the singularity in $V(\mathbf{U})$ corresponds to the disjointness in $\tilde{R}$.

For $\mathbf{U} \in \mathfrak{M}_{s}(R)$ by (2.2) $V(\mathbf{U})$ is isomorphic to the completion of $(R, \mathbf{U}) / N(\mathbf{U})$; specially for $\mathbf{U}=\mathbf{U}_{s}$ we get from (2.2) that $\tilde{R}, V\left(\tilde{\mathbf{U}}_{s}\right)$ and $V\left(\mathbf{U}_{s}\right)=\mathfrak{M}_{s}(R)$ are isomorphic.

(2.5) Corollary. $\mathfrak{M}_{s}(R)$ is a complete Boolean algebra. 
It's a reasonable question and of interest in connection with the Lebesgue decomposition of contents, whether the lattice of all locally $s$-bounded monotone ring topologies on $R$ (for the definition s.e.g. [24]) also forms a Boolean algebra; this, however, isn't true:

(2.6) COROLlaRY. For $\mathrm{U} \in \mathfrak{M}(R)$ the following statements are equivalent:

(1) $\mathbf{U}$ is s-bounded.

(2) $V(\mathbf{U})$ is a complete Boolean algebra.

(3) For every $\mathbf{V} \in \mathfrak{M}(R)$ there are uniquely determined $\mathbf{U}_{1}, \mathbf{U}_{2} \in \mathfrak{M}(R)$ such that $\mathbf{U}=\mathbf{U}_{1} \vee \mathbf{U}_{2}, \mathbf{U}_{1} \subset \mathbf{V}$ and $\mathbf{U}_{2}, \mathbf{V}$ are singular.

(4) $V(\mathbf{U})$ is a complemented lattice.

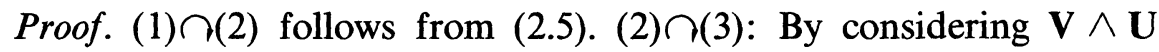
instead of $\mathbf{V}$ the assertion in reduced to the case that $\mathbf{V} \in V(\mathbf{U})$. In this case the unique decomposition of $\mathbf{U}$ in the sense of (3) is yielded directly by (2).

(3)ก(4) is obvious.

(4) (1): If $\mathbf{U}$ is not $s$-bounded, then, for some $U \in \mathbf{U}$, there is a disjoint sequence $\left(a_{n}\right)$ in $R \backslash U$. Let $U_{0}$ be a normal set from $U$ with $U_{0} \vee U_{0} \subset U$. For $\mathbf{W}:=\sup _{n} p_{\mathbf{U}}\left(a_{n}\right)$ there exists by (4) a topology $\mathbf{V} \in$ $V(\mathbf{U})$ such that $\mathbf{U}=\mathbf{V} \vee \mathbf{W}$ and $\mathbf{V}, \mathbf{W}$ are singular. Since $\mathbf{U}=\mathbf{V} \vee \mathbf{W}$ there is a normal set $V \in \mathbf{V}$ and sets $U_{1}, \ldots, U_{m} \in \mathbf{U}$ with $U_{0} \supset V \cap$ $\cap_{i=1}^{m}\left(U_{i} \wedge a_{i}\right) \vee\left(R \backslash a_{i}\right) \supset V \wedge a_{m+1}$. Because of the singularity of $\mathbf{V}, \mathbf{W}$ there are elements $v \in V$ and $w \in\left(U_{0} \wedge a_{m+1}\right) \vee\left(R \backslash a_{m+1}\right)$ with $a_{m+1}$ $=v \vee w$. Then $v \in V \wedge a_{m+1} \subset U_{0}, w \in U_{0} \wedge a_{m+1} \subset U_{0}$, hence $a_{m+1}$ $\in U_{0} \vee U_{0} \subset U$, and so we get a contradiction.

The existence statement of (3) for $U \in \mathfrak{M}_{s}(R)$ was first proved by Traynor [21, Chapter 4], who so could answer a question of Drewnowski $[5$, p. 47]. Specially choosing the discrete topology for $U$ the equivalence $(1) \cap(4)$ of (2.6) yields: $\mathfrak{M}(R)$ is a complemented lattice iff $R$ is finite. (In this case $\mathfrak{M}(R)$ and $R$ are isomorphic.)

(2.7) Corollary. For a Hausdorff topology $\mathbf{U} \in \mathfrak{M}(R)$ the following statements are equivalent:

(1) $\mathbf{U}$ is s-bounded and complete.

(2) $(R, \leq)$ is complete and $\mathbf{U} \tau$-smooth.

(3) $p_{\mathbf{U}}: R \rightarrow V(\mathbf{U})$ is a lattice isomorphism.

(4) $p_{\mathbf{U}}: R \rightarrow V(\mathbf{U})$ is surjective. 


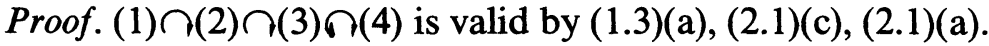

(3) (1): By (3) $V(\mathbf{U})$ is a Boolean algebra, so by (2.6) $\mathbf{U}$ is $s$-bounded. $\mathrm{U}$ is complete: With the notation of (2.2) by (2.2)(c) $\pi^{-1} \circ p_{\mathrm{U}}: R \rightarrow \tilde{R}$ is surjective. For $v \in R \subset \tilde{R}$ the convergence to 0 of a net $\left(x_{\alpha}\right)$ in $R$ with respect to $p_{\mathbf{U}}(v)$ as well as with respect to $p_{\tilde{\mathbf{U}}}(v)$ just means that $\left(x_{\alpha} \wedge v\right)$ converges to 0 with respect to $\mathrm{U}$; so $p_{\mathrm{U}}(v)=p_{\tilde{\mathbf{U}}}(v) \mid R=\pi(v)$ and $\left(\pi^{-1} \circ p_{\mathrm{U}}\right)(v)=v$. Hence $R=\tilde{R}$ and so $\mathrm{U}$ is complete.

We now compare $(2.3)(\mathrm{a}),(2.7)(1) \cap(2)$ with [12; Theorems 4.2, 4.3, 4.5 of Chapter III]. In [12] in a much more special way than here monotone ring topologies on a $\sigma$-algebra of sets are considered, which are always generated by a system of real-valued nonnegative measures. [12, Theorem III.4.5] is a special case of (2.3)(a). Also [12, Theorm IV.7.2] stating that every indefinite integral with respect to a closed vector

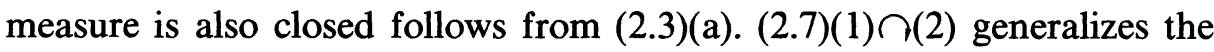
first statement of [12, Theorem III.4.2] and (2.7)(2)へ(1) [12, Theorem III.4.3]. Instead of the $\tau$-smoothness in (2.7)(2) the assumption in [12, Theorem III.4.3] is that no disjoint set of the quotient space of the $\sigma$-algebra by the ideal of nullsets has measurable cardinal; this cardinal number condition implies in the special situation considered in [12] by [12, Lemma III.4.1] the $\tau$-smoothness.

Here is a generalization of the "Lebesgue decomposition" for $\mathbf{U} \in$ $\mathfrak{M}_{s}(R)$ established in $(2.6)(3)$ :

(2.8) CoRollary. Let be $\mathbf{V} \in \mathfrak{M}_{s}(R)$ and $\left(\mathbf{U}_{\alpha}\right)_{\alpha \in A}$ a family in $\mathfrak{M}(R)$. Then there are $\mathbf{V}_{\alpha}, \mathbf{W} \in \mathfrak{M}_{s}(R)(\alpha \in A)$, such that $\mathbf{V}=\mathbf{W} \vee \sup _{\alpha \in A} \mathbf{V}_{\alpha}$, for all $\alpha \in A, \mathbf{V}_{\alpha} \subset \mathbf{U}_{\alpha}$ and $\mathbf{W}, \mathbf{U}_{\alpha}$ are singular and the $\mathbf{V}_{\alpha}$ are pairwise singular. Here $\mathbf{W}$ and $\sup _{\alpha \in A} \mathbf{V}_{\alpha}$ are uniquely determined. If the $\mathbf{U}_{\alpha}$ are pairwise singular, then the $\mathbf{V}_{\alpha}$ are uniquely determined, too.

Proof. We may assume that the $\mathbf{U}_{\alpha}$ are $s$-bounded; otherwise consider $\mathbf{V} \wedge \mathbf{U}_{\alpha}$ instead of $\mathbf{U}_{\alpha}$. In the $s$-bounded case (2.8) follows from (2.5). A corresponding statement is true in every complete Boolean algebra, you know. This becomes clear noting the statement $[19,20.2]$, which will be formulated in (2.9)(a) specially for $\mathfrak{M}_{s}(R)$.

(2.9) Proposition. (a) For every family $\left(\mathbf{U}_{\alpha}\right)_{\alpha \in A}$ in $\mathfrak{M}_{s}(R)$ there is a family $\left(\mathbf{V}_{\alpha}\right)_{\alpha \in A}$ of pairwise singular, s-bounded monotone ring topologies on $R$ with $\sup _{\alpha \in A} \mathbf{U}_{\alpha}=\sup _{\alpha \in A} \mathbf{V}_{\alpha}$ and $\mathbf{V}_{\alpha} \subset \mathbf{U}_{\alpha}(\alpha \in A)$.

(b) For every $\mathbf{V} \in \mathfrak{M}_{s}(R)$ there is a family $\left(\mathbf{V}_{\alpha}\right)_{\alpha \in A}$ of pairwise singular, s-bounded monotone ring topologies, each of which possesses an at most countable 0-neighbourhood base, with $\mathbf{V}=\sup _{\alpha \in A} \mathbf{V}_{\alpha}$. 
Proof. (a) follows from (2.5) and [19, 20.2].

(b) Every $\mathbf{V} \in \mathfrak{M}_{s}(R)$ is the supremum of a family $\left(\mathrm{U}_{\alpha}\right)_{\alpha \in A}$ in $\mathfrak{M}_{s}(R)$, each of which possesses an at most countable 0 -neighbourhood base. We then choose $\left(\mathbf{V}_{\alpha}\right)_{\alpha \in A}$ for $\left(\mathbf{U}_{\alpha}\right)_{\alpha \in A}$ according to (a) and observe that for every $\alpha \in A$ by (2.3)(b) also $V_{\alpha}$ has an at most countable 0 -neighbourhood base.

For an arbitrary cardinal number $\kappa$ a Boolean ring is said to satisfy the $\kappa$-chain condition provided every set of disjoint elements in it has power $\leq \kappa$. The proof of the following theorem is also essentially based on the isomorphism established in (2.2)(c).

(2.10) ThEOREM. For $\mathrm{U} \in \mathfrak{M}_{s}(R)$ and an infinite cardinal number $\kappa$ the following statements are equivalent:

(1) U possesses a 0 -neighbourhood base of power $\leq \kappa$.

(2) The completion of $(R, \mathbf{U}) / N(\mathbf{U})$ satisfies the $\kappa$-chain condition.

(3) $V(\mathbf{U})$ satisfies the $\kappa$-chain condition.

Proof. Let $(\tilde{R}, \tilde{\mathbf{U}})$ be the completion of the quotient space $(\hat{R}, \hat{\mathbf{U}}):=(R, \mathbf{U}) / N(\mathbf{U})$. Since $V(\mathbf{U})$ and $V(\hat{\mathbf{U}})$ are evidently isomorphic (s. [24, (19)]) and by $(2.2)($ c) $V(\hat{\mathbf{U}})$ and $\tilde{R}$ are isomorphic, (2) and (3) are equivalent.

(3) (1): By (2.9)(b) there are pairwise singular $\mathbf{U}_{\alpha} \in \mathfrak{M}_{s}(R)$, each of which has an at most countable basis $\left(U_{\alpha, n}\right)_{n \in \mathbf{N}}(\alpha \in A)$, with $\mathbf{U}=$ $\sup _{\alpha \in A} \mathbf{U}_{\alpha}$.

Since by (3) $|A| \leq \kappa$ the subbase $\left\{U_{\alpha, n}: \alpha \in A, n \in \mathbf{N}\right\}$ of $\mathbf{U}$ has power $\leq \kappa$. So $U$ has also a base of power $\leq \kappa$.

$(1) \cap(2)$ : Since with $\mathbf{U}$ also $\tilde{U}$ possesses a base of power $\leq \kappa$ we may assume that $\mathbf{U}$ is Hausdorff and complete. Let $\left(U_{\alpha}\right)_{\alpha \in A}$ be a base of $\mathbf{U}$ of power $\leq \kappa$ and $D$ a disjoint subset of $R$. Since $U$ is Hausdorff $D=$ $\cup_{\alpha \in A}\left(D \backslash U_{\alpha}\right)$. Because of the $s$-boundedness of $\mathbf{U}$ each of the sets $D \backslash U_{\alpha}$ is finite, hence $|D| \leq \kappa$.

From the equivalence $(1) \cap(3)$ we get $(2.3)(b)$ once again.

(2.11) Corollary. Assume for an infinite cardinal number $\kappa R$ is $\kappa$-complete, $\mathrm{U}$ a $\kappa$-smooth monotone ring topology on $R$. Then the following statements are equivalent:

(1) $\mathbf{U}$ possesses a 0 -neighbourhood base of power $\leq \kappa$.

(2) $R / N(\mathbf{U})$ satisfies the $\kappa$-chain condition.

If (1), (2) hold, $\mathbf{U}$ is complete, the quotient topology on $R / N(\mathbf{U})$ induced by $\mathbf{U}$ is $\tau$-smooth and $R / N(\mathbf{U})$ is complete as lattice. 
Proof. In view of (1.2) we may assume that $\mathbf{U}$ is Hausdorff. By (2.10) (2) follows from (1). Now assume (2). By [19, 20.5] $R$ is complete. We repeat the proof from [19] because it also yields the $\tau$-smoothness of $U$ : Let $M$ be an increasingly directed subset of $R$. With $[19,20.2]$ it is easy to see that $M$ has a cofinal subset $M_{0}$ of power $\leq \kappa$. So there is an element $a \in R$ with $a=\sup M_{0}=\sup M, M_{0}$ converges to $a$ with respect to $\mathrm{U}$ and so $M$ converges to $a$, too. Now it follows by $(2.7)(2) \cap(1)$ that $\mathbf{U}$ is complete and by (2.10) that $\mathrm{U}$ has a base of power $\leq \kappa$.

Drewnowski [6, Theorem 1.2] proved the equivalence (1)凡(2) of (2.11) in case that $\kappa$ is countable and $R$ is a $\sigma$-ring of sets.

As in [24] we call a monotone ring topology $\mathbf{U}$ on $R$ atomless (atomar) if the completion of $(R, U) / N(\mathrm{U})$ is an atomless (atomar) Boolean ring, respectively. In accordance with $[14,2.3,2.4]$ we call $\mathbf{U}$ an ultrafilter topology, if $N(\mathbf{U})$ is a maximal ideal in $R$. If $N$ is a maximal ideal in $R$, $\mu(x)=0(x \in N)$ and $\mu(x)=1(x \in R \backslash N)$ defines a content $\mu: R \rightarrow$ $\left[0, \infty\left[; \mathbf{W}_{0}(\mu)=\{U: N \subset U \subset R\}\right.\right.$ is the only topology $\mathbf{U} \in \mathfrak{M}(R)$ with $N(\mathbf{U})=N$; since $|R / N|=2, \mathbf{W}_{0}(\mu)$ is complete, atomar and $s$-bounded. For an illustration we note that for a non-empty subset $N$ of $R$ the following statements are equivalent, as well-known (for definitions s. [19]):

(1) $N$ is a maximal ideal in $R$.

(2) $N$ is an ideal in $R$ and $|R / N|=2$.

(3) $N$ is an ideal in $R$ and $\{x \in R: x \notin N\}$ is a filter.

(4) $\{x \in R: x \notin N\}$ is an ultrafilter.

If $R$ is a Boolean algebra, a further equivalence is

(5) $\{e \backslash x: x \in N\}$ is an ultrafilter.

In the following we clarify in some cases, which properties of topologies from $V(\mathbf{U})$ and of elements from $\tilde{R}$ correspond each other by the isomorphism established in (2.2)(c).

(2.12) Proposition. By the same assumptions and notations as in (2.2) let be $\mathbf{V} \in V(\mathbf{U})$ and $v \in \tilde{R}$ with $\pi(v)=\mathbf{V}$.

(a) For an infinite cardinal number $\kappa \mathbf{V}$ has a 0-neighbourhood base of power $\leq \kappa$ iff $\tilde{R} \wedge v$ satisfies the $\kappa$-chain condition.

(b) $\mathbf{V}$ is atomless (atomar) iff $\tilde{R} \wedge v$ is atomless (atomar) (i.e. the Boolean algebra $V(\mathbf{V})$ is atomless (atomar)), respectively.

(c) $\mathbf{V}$ is an ultrafilter topology iff $v$ is an atom of $\tilde{R}$ (i.e. $\mathbf{V}$ is an atom of the Boolean algebra $V(\mathbf{U})$ ).

Proof. Use that by $(2.2)(\mathrm{d})$ the completion of $(R, \mathbf{V}) / N(\mathbf{V})$ and $(\tilde{R} \wedge v, \tilde{\mathbf{U}} \mid \tilde{R} \wedge v)$ are isomorphic. From this follows (b) and by (2.10) 
also (a). For the proof of (c) check the equivalence of the following statements one after the other: $\mathbf{V}$ is an ultrafilter topology, $|R / N(\mathbf{V})|=2$, $|\tilde{R} \wedge v|=2, v$ is an atom of $\tilde{R}$.

(2.13) CoRollary. (a) The set of all atomless topologies from $\mathfrak{M}_{s}(R)$ and the set of all atomic topologies from $\mathfrak{M}_{s}(R)$ each form a complete ideal in $\mathfrak{M}_{s}(R) . \mathfrak{M}_{s}(R)$ is the direct sum of these ideals.

(b) $\mathbf{U} \in \mathfrak{M}(R)$ is $s$-bounded and atomar iff $\mathbf{U}$ is the supremum of a family of ultrafilter topologies on $R$.

Proof. Any complete Boolean algebra is the direct product of an atomless and an atomic complete Boolean algebra; further the unit of an atomic Boolean algebra is the supremum of its atoms. Translate this by the isomorphism of (2.2)(c) observing (2.12).

(2.13) (a) we proved in [24] in a different way. By (2.13)(a) every $\mathbf{U} \in \mathfrak{M}_{s}(R)$ can be written in the form $\mathbf{U}=\mathbf{U}_{a} \vee \mathbf{U}_{l}$ with an atomic $\mathbf{U}_{a} \in \mathfrak{M}_{s}(R)$ and an atomless $\mathbf{U}_{l} \in \mathfrak{M}_{s}(R)$; if $\mathbf{U}_{a}$ is further decomposed according to (2.13)(b) and $U_{l}$ according to (2.9)(b), so we get:

(2.14) CoROllaRY. For every $\mathbf{U} \in \mathfrak{M}_{s}(R)$ there is a family $\left(\mathbf{U}_{\alpha}\right)_{\alpha \in A}$ of ultrafilter topologies in $\mathfrak{M}_{s}(R)$ and a family $\left(\mathbf{U}_{\beta}\right)_{\beta \in B}$ of atomless topologies in $\mathfrak{M}_{s}(R)$, each of which possesses a countable 0-neighbourhood base, such that $\mathbf{U}=\left(\sup _{\alpha \in A} \mathbf{U}_{\alpha}\right) \vee\left(\sup _{\beta \in B} \mathbf{U}_{\beta}\right)$ and the $\mathbf{U}_{\gamma}(\gamma \in A \cup B)$ are pairwise singular.

Given a decomposition for $U \in \mathfrak{M}_{s}(R)$ in the sense of (2.14), then $\mathbf{V}=\sup _{\alpha \in A}\left(\mathbf{U}_{\alpha} \wedge \mathbf{V}\right) \vee \sup _{\beta \in B}\left(\mathbf{U}_{\beta} \wedge \mathbf{V}\right)$ is the corresponding decomposition for $\mathbf{V} \in V(\mathbf{U})$; this is easy to see by (2.5), (2.3)(b), (2.12)(c), (2.13)(a). If $\mathbf{U} \in \mathfrak{M}_{s}(R)$ is Hausdorff and complete, then decompositions of $\mathbf{U}$ correspond to decompositions of $R$ according to (2.2)(c). The decomposition given in (2.14) corresponds to the following:

(2.15) THEOREM. If $\mathbf{U} \in \mathfrak{M}_{s}(R)$ is Hausdorff and complete, then $(R, \mathbf{U})$ is topological isomorphic to a product $\left(2^{A}, \mathbf{U}_{p}\right) \times \Pi_{\beta \in B}\left(R_{\beta}, \mathbf{U}_{\beta}\right)$, where $\mathbf{U}_{p}$ is the product topology on $2^{A}=\{0,1\}^{A},\{0,1\}$ endowed with the discrete topology, and, for $\beta \in B, \mathbf{U}_{\beta}$ is a metrizable, atomless, $\tau$-smooth monotone ring topology on a complete Boolean algebra satisfying CCC.

Proof. Starting from the decomposition of $\mathbf{U}$ given in (2.14) $x_{\alpha}:=\pi^{-1}\left(\mathbf{U}_{\alpha}\right)(\alpha \in A)$ are all atoms of $R, \mathbf{U} \mid R \wedge x_{\alpha}$ is the discrete 
topology; for $x_{\beta}:=\pi^{-1}\left(\mathbf{U}_{\beta}\right)(\beta \in B) R_{\beta}:=R \wedge x_{\beta}$ satisfies CCC by (2.12)(a), by the definition of $\pi$ we have $\mathrm{U}\left|R \wedge x_{\beta}=\mathbf{U}_{\beta}\right| R \wedge x_{\beta}$ and these topologies are metrizable, atomless and by (2.2)(b) $\tau$-smooth. Further $x_{\gamma}(\gamma \in A \dot{\cup} B)$ are pairwise disjoint elements with $\sup _{\gamma} x_{\gamma}=e$. The assertion is now a consequence of the following lemma.

(2.16) LEMMA. If $\mathbf{U} \in \mathfrak{M}_{s}(R)$ is Hausdorff and complete and $\left(x_{\gamma}\right)_{\gamma \in \Gamma}$ is a disjoint family in $R$ with $\sup _{\gamma} x_{\gamma}=e$, then $x \mapsto\left(x \wedge x_{\gamma}\right)_{\gamma \in \Gamma}$ is a topological isomorphism from $(R, \mathbf{U})$ onto $\Pi_{\gamma \in \Gamma}\left(R \wedge x_{\gamma}, \mathbf{U} \mid R \wedge x_{\gamma}\right)$.

Proof. Since $(R, \leq)$ is complete by (1.3)(a), the given mapping is an algebraic isomorphism. That this is also a topological mapping, follows from $\mathbf{U}=\sup _{\gamma} p_{\mathbf{U}}\left(x_{\gamma}\right)(\mathrm{s.}(2.2)(\mathrm{c}))$.

If there exists a complete, $s$-bounded, Hausdorff monotone ring topology on $R$, then by (2.15) $R$ is the product of complete Boolean algebras, each of which satisfies CCC. (This is not true for every complete Boolean algebra.) From this we also get that $R$ contains a disjoint subset of maximal power. On the other hand, if one assumes the existence of uncountable regular cardinal numbers, there are complete Boolean algebras, which contain no disjoint subset of maximal power (s. [7]).

3. Decomposition of finitely additive set functions. Now we give a new, simple proof for decomposition theorems of contents based on theorem (2.2). Here the structure of the range space is of subordinate importance. To emphasize this we choose the range space in the following generality (compare [24, Chapter 6]). Throughout this chapter let

$(G, \mathbf{W})$ be a separated uniform space, $\mathbf{W}$ its uniformity,

$+:(G, \mathbf{W}) \times(G, \mathbf{W}) \rightarrow(G, \mathbf{W})$ a uniformly continuous operation, $\mu: R \rightarrow G$ a content $($ i.e. $\mu(a \vee b)=\mu(a)+\mu(b)$ for disjoint $a, b \in R)$.

$\mathbf{W}(\mu)$ stands for the coarsest monotone ring topology on $R$ such that $\mu:(R, \mathbf{W}(\mu)) \rightarrow(G, \mathbf{W})$ is continuous; then $\mu$ is even uniformly continuous. With $0:=\mu(0) N(\mu):=N(\mathbf{W}(\mu))=\{a \in R: \mu(R \wedge a)=\{0\}\}$ is the system of " $\mu$-null sets"; the sets $\left\{a \in R: \mu(R \wedge a) \subset W_{0}\right\}$, where $W_{0}$ runs through the neighbourhood system of $\mu(0)$, from a 0 -neighbourhood base of $\mathbf{W}(\mu) . \mu$ is called $s$-bounded, $\kappa$-smooth, atomless, atomic (with respect to $\mathbf{W})$, iff $\mathbf{W}(\mu)$ is $s$-bounded, $\kappa$-smooth, atomless, atomic, respectively. It is easy to see that $\mu$ is $s$-bounded iff for each disjoint sequence $\left(a_{n}\right)$ in $R\left(\mu\left(a_{n}\right)\right)$ converges to 0 . An analogic transformation of $\kappa$-smoothness is possible; for a somewhat more general statement see lemma (3.6.2). 
For $\mathbf{U} \in \mathfrak{M}(R) \mu$ is called $\mathbf{U}$-continuous (with respect to $\mathbf{W}$ ), if $\mathbf{W}(\mu) \subset \mathbf{U}$, i.e. if $\mu:(R, \mathbf{U}) \rightarrow(G, \mathbf{W})$ is continuous, and $\mathbf{U}$-singular (with respect to $W$ ), if $W(\mu)$ and $U$ are singular. If $\mu^{\prime}$ is another content with values in a separated uniform space $\left(G^{\prime}, \mathbf{W}^{\prime}\right)$ carrying a uniformly continuous operation $+:\left(G^{\prime}, \mathbf{W}^{\prime}\right) \times\left(G^{\prime}, \mathbf{W}^{\prime}\right) \rightarrow\left(G^{\prime}, \mathbf{W}^{\prime}\right), \mu$ and $\mu^{\prime}$ are called singular (written $\mu \perp \mu^{\prime}$ ), if $\mathbf{W}(\mu)$ and $\mathbf{W}\left(\mu^{\prime}\right)$ are singular. All decompositions of $\mu$ given in the following are produced by a decomposition of the completion of $\left(R, \mathbf{U}_{s}\right)$.

(3.1) Proposition. Let $\mu$ be s-bounded, $(G, \mathbf{W})$ complete, $\left(\tilde{R}, \tilde{\mathbf{U}}_{s}\right)$ the completion of $\left(R, \mathbf{U}_{s}\right)$ and $u \in \tilde{R}$.

(a) Then there is a unique continuous extension $\tilde{\mu}:\left(\tilde{R}, \tilde{\mathbf{U}}_{s}\right) \rightarrow(G, \mathbf{W})$ of $\mu ; \tilde{\mu}$ is a s-bounded content and $\mathbf{W}(\tilde{\mu}) \mid R=\mathbf{W}(\mu)$.

(b) The maps $\lambda$ and $\mu$ defined by $\lambda(a):=\tilde{\mu}(a \backslash u), \nu(a):=\tilde{\mu}(a \wedge u)$ $(a \in R)$ are $G$-valued, s-bounded contents on $R$ with the following properties: $\mu=\lambda+\nu=\nu+\lambda, \lambda(0)=\nu(0)=0$;

$\lambda(R), \nu(R) \subset \tilde{\mu}(\tilde{R}) \subset \overline{\mu(R)}$;

$\mathbf{W}(\mu)=\mathbf{W}(\lambda) \vee \mathbf{W}(\nu) ; \lambda \perp \nu$.

(c) If by the notation of (2.2) (with $\mathbf{U}:=\mathbf{U}_{s}$ ) we choose $m \in \tilde{R}$ with $\pi(m)=\mathbf{W}(\mu)$, then $\pi(m \backslash u)=\mathbf{W}(\lambda), \pi(m \wedge u)=\mathbf{W}(\nu)$ and $\tilde{\mu}(a)=\tilde{\mu}(a$ $\wedge m)$ for all $a \in \tilde{R}$.

Proof. (a) The $s$-boundedness of $\mu$ implies $\mathbf{W}(\mu) \subset \mathbf{U}_{s}$, so $\mu$ : $\left(R, \mathbf{U}_{s}\right)$ $\rightarrow(G, \mathbf{W})$ is uniformly continuous and has a unique continuous extension $\tilde{\mu}:\left(\tilde{R}, \tilde{\mathbf{U}}_{s}\right) \rightarrow(G, \mathbf{W})$; with $\mu$ also $\tilde{\mu}$ is finitely additive. Since $\mathbf{W}(\tilde{\mu}) \subset \tilde{\mathbf{U}}_{s}$ and $\tilde{\mathbf{U}}_{s}$ is $s$-bounded by (2.2)(a), $\tilde{\mu}$ is also $s$-bounded. It is easy to see and follows from [24, (6.2)] that $\mathbf{W}(\tilde{\mu}) \mid R=\mathbf{W}(\mu)$.

(b), (c). Obviously $\tilde{\lambda}(a):=\tilde{\mu}(a \backslash u)$ and $\tilde{\nu}(a):=\tilde{\mu}(a \wedge u)(a \in \tilde{R})$ define $s$-bounded contents on $\tilde{R}$ with values in $\tilde{\mu}(\tilde{R})(\subset \overline{\mu(R)})$ and $\tilde{\mu}=\tilde{\lambda}+\tilde{\nu}=\tilde{\nu}+\tilde{\lambda}, \tilde{\lambda}(0)=\tilde{\nu}(0)=0$ : similar statements are true for $\lambda$ and $\nu$. Since $\mathbf{W}(\tilde{\nu}) \mid R=\mathbf{W}(\nu)$ (s. (a)) and $e \backslash \sup N(\tilde{\nu})=(e \backslash \sup N(\tilde{\mu})) \wedge u=$ $m \wedge u$, we have $\pi(m \wedge u)=\mathbf{W}(\nu)($ s. $(2.1)(\mathrm{b}),(2.2)(\mathrm{c}))$; similarly $\pi(m \backslash u)$ $=\mathbf{W}(\lambda)$. Because $\pi$ is a lattice isomorphism, the equalities $m=(m \backslash u) \vee$ $(m \wedge u)$ and $(m \backslash u) \wedge(m \wedge u)=0$ just mean that $\mathbf{W}(\mu)=\mathbf{W}(\lambda) \vee$ $\mathbf{W}(\nu)$ and $\mathbf{W}(\lambda), \mathbf{W}(\nu)$ are singular. Finally, $e \backslash m=\sup N(\tilde{\mu})$ implies $\tilde{\mu}(a)=\tilde{\mu}(a \wedge m)+\tilde{\mu}(a \backslash m)=\tilde{\mu}(a \wedge m)+0=\tilde{\mu}(a \wedge m)$ for all $a \in \tilde{R}$.

(3.2) Corollary. Assume $(G, \mathbf{W})$ is complete and $\left(\tilde{R}, \tilde{\mathbf{U}}_{s}\right)$ the completion of $\left(R, \mathbf{U}_{s}\right)$. For any $s$-bounded content $\nu: R \rightarrow G$ denote by $\tilde{\nu}$ the

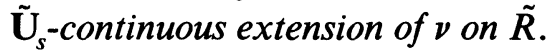


Then $\nu \leftrightarrow \tilde{\nu}$ defines a bijection between the set of all G-valued, $s$-bounded contents on $R$ and the set of all G-valued, $\tau$-smooth contents on $\tilde{R}$. Moreover, if $(G,+, \mathbf{W})$ is a commutative topological group, $\nu \leftrightarrow \tilde{\nu}$ defines a group isomorphism.

This follows from (3.1)(a) and (2.2)(b).

(3.2) clears the connection between our technic and Stone space arguments mentioned in the introduction.

Now let's illustrate the method of (3.1) for decomposing of contents by the Jordan decomposition. Suppose, $\mu$ is a real-valued content, $\tilde{R}, \tilde{\mathbf{U}}_{s}, \tilde{\mu}$ as in (3.1), $P:=\{a \in \tilde{R}: \tilde{\mu}(\tilde{R} \wedge a) \subset[0, \infty[\}$ and for $u:=\sup P \lambda$ and $\nu$ are chosen as in (3.1). Then $\mu=\lambda+\nu, \nu \geq 0$, and - as can be shown by usual arguments $-\lambda \leq 0$. So the Hahn decomposition of $\tilde{R}$ (or $\tilde{\mu}$ ) yields the Jordan decomposition of $\mu$.

The following Lebesgue decomposition was proved (in the group-valued case) by Traynor [21, Theorem 1.2'] using longer technical arguments. His theorem, which answered a question of Drewnowski [5, p. 47], is here an immediate consequence of (3.1).

(3.3) Theorem. Let $\mu$ be s-bounded, $(G, \mathbf{W})$ complete and $\mathbf{U} \in \mathfrak{M}(R)$. Then there exist unique G-valued, s-bounded contents $\lambda$ and $\nu$ on $R$ such that $\lambda$ is $\mathbf{U}$-singular, $\nu$ is $\mathbf{U}$-continuous, $\mu=\lambda+\nu$ and $\lambda(0)=\nu(0)=0$.

Moreover: $\mathbf{W}(\mu)=\mathbf{W}(\lambda) \vee \mathbf{W}(\nu), \lambda \perp \nu, \lambda+\nu=\nu+\lambda, \lambda(R) \cup$ $\nu(R) \subset \overline{\mu(R)}$.

Proof. We may assume that $\mathbf{U}$ is $s$-bounded; otherwise replace $\mathbf{U}$ by $\mathbf{U} \wedge \mathbf{U}_{s}$. We use the notations $\tilde{R}, \tilde{\mathbf{U}}_{s}, \tilde{\mu}, \pi, m$ as in (3.1). $\lambda$ and $\nu$ chosen for $u:=\pi^{-1}(\mathbf{U})$ as in (3.1) satisfy the desired properties. (Observe that by (3.1)(c), (2.2)(c) $(m \backslash u) \wedge u=0$ and $m \wedge u \leq u$ just mean that $\lambda$ is $\mathbf{U}$-singular and $\nu \mathbf{U}$-continuous.)

For the proof of the uniqueness assertion let $\lambda$ and $\nu$ are as stated. Then the $\tilde{\mathbf{U}}_{s}$-continuous extensions $\tilde{\lambda}, \tilde{\nu}$ of $\lambda, \nu$ on $\tilde{R}$ are contents with $\tilde{\mu}=\tilde{\lambda}+\tilde{\nu}$. If $l, n \in \tilde{R}$ with $\pi(l)=\mathbf{W}(\lambda)$ and $\pi(n)=\mathbf{W}(\nu)$, the U-singularity of $\lambda$ and the $\mathrm{U}$-continuity of $\nu$ just mean that $l \wedge u=0$ and $n \leq u$ (s. (2.2)(c)). Since by $(3.1)(\mathrm{c}) \tilde{\lambda}(x)=\tilde{\lambda}(x \wedge u), \tilde{\nu}(x)=\tilde{\nu}(x \wedge n)$ for $x \in \tilde{R}$, we get for $a \in R$

$$
\begin{aligned}
\tilde{\mu}(a \wedge u) & =\tilde{\lambda}(a \wedge u)+\tilde{\nu}(a \wedge u)=\tilde{\lambda}(a \wedge u \wedge l)+\tilde{\nu}(a \wedge u \wedge n) \\
& =\tilde{\lambda}(0)+\tilde{\nu}(a \wedge n)=0+\tilde{\nu}(a)=\nu(a),
\end{aligned}
$$

similarly $\tilde{\mu}(a \backslash u)=\lambda(a)$. This finishes the proof. 
By the notation of this proof there exists a Cauchy net $\left(x_{\gamma}\right)_{\gamma \in \Gamma}$ in $(R, \mathbf{W}(\mu))$, such that $\left(x_{\gamma} \wedge m\right)_{\gamma \in \Gamma}$ converges with respect to $\tilde{\mathbf{U}}_{s}$ to $n$ $(\in \tilde{R} \wedge m)$ (s. (2.2)(d)); using (3.1)(c) we get

$$
\nu(a)=\tilde{\mu}(a \wedge n)=\lim _{\gamma} \tilde{\mu}\left(a \wedge x_{\gamma} \wedge m\right)=\lim _{\gamma} \mu\left(a \wedge x_{\gamma}\right) \quad(a \in R) .
$$

If $(R, \mathbf{W}(\mu))$ is complete, one can choose $x_{\gamma}=n_{0}(\gamma \in \Gamma)$ for some $n_{0} \in R$ and so $\nu(a)=\mu\left(a \wedge n_{0}\right)$. An analogic representation of $\lambda$ is valid. So we have proved:

(3.4) REMARK. Instead of the completeness of $(G, \mathbf{W})$ it is enough to assume in (3.3) that each Cauchy net of form $\left(\mu\left(z_{\gamma}\right)\right)_{\gamma \in \Gamma}$, where $\left(z_{\gamma}\right)_{\gamma \in \Gamma}$ is a Cauchy net in $(R, \mathbf{W}(\mu))$, converges in $(G, \mathbf{W})$.

That is fulfilled, if $(R, \mathbf{W}(\mu))$ is complete; in this case there are disjoint elements $l, n \in R$ such that $\lambda(a)=\mu(a \wedge l), \nu(a)=\mu(a \wedge n)$ for all $a \in R$.

The last statement could also be seen directly, if we would work in the proof of (3.3) with the completion of $(R, \mathbf{W}(\mu)) / N(\mu)$ instead of $\left(\tilde{R}, \tilde{\mathbf{U}}_{s}\right)$.

The relation $\mathbf{W}(\mu)=\mathbf{W}(\lambda) \vee \mathbf{W}(\nu)$ in (3.3) has the following significance: It implies e.g. that $\mu$ is "regular" iff both $\lambda$ and $\nu$ are "regular" (for definition s. (3.6)(a)); further that $\mu$ is $\kappa$-smooth iff both $\lambda$ and $\nu$ are $\kappa$-smooth. If $\mu$ is a $\sigma$-additive set function, so $\lambda$ and $\nu$, too; a further assumption to $\mathbf{U}$ as supposed in [22, Theorem 5] can be dropped. Uhl proved in [22, Theorem 5] the Lebesgue decomposition of Banach spacevalued contents in case that the topology $U$ of (3.3) is induced by a nonnegative real-valued content; using Stone space arguments he reduced the finitely additive case to the $\sigma$-additive one; he assumed instead of the $s$-boundedness the equivalent condition that $\mu$ has a $[0, \infty[$-valued "control content" or equivalently that $\mu$ has a relatively weakly compact range; (of course, the assumption (3) in [22, Theorem 5] is not - as in [22] noted - an equivalent condition); for the notion of singularity of [22] $\mathrm{s}$. (3.7).

The decompositions according to (3.1)(b) are just the decompositions according to (3.3); the connection between $u$ and $\mathrm{U}$, appearing in (3.1)(b) and (3.3) respectively, is $\pi(u)=\mathbf{U}(\pi$ as in (3.1)(c)).

For a comparison we now describe all decompositions, which can be obtained by ideals in $R$ with the method of Traynor described in [20]. The decompositions according to [20, Theorem 1.7] exactly correspond to those decompositions according to $(3.1)(\mathrm{b})$, which are obtained by elements $u \in \tilde{R}$ of form $u=\sup M, M \subset R$, sup built in $\tilde{R}$. (Observe that 
two elements $u_{1}, u_{2} \in \tilde{R}$ yield the same decomposition according to (3.1) iff $m \wedge u_{1}=m \wedge u_{2}$ or $m \wedge u_{1}=m \backslash u_{2}$.) Now we give an example for a decomposition, which can be obtained by (3.1) (or (3.3) or [21, Theorem 1.2]), but not by [20, Theorem 1.7]: Let $R$ be the ring generated by the intervals of form $[\alpha, \beta[, 0 \leq \alpha<\beta \leq 1, G$ the linear space of all real-valued functions on $[0,1$ [ endowed with the topology of pointwise convergence and for $a \in R \mu(a):=\chi_{a}$ the characteristic function of $a$ defined on $\left[0,1\left[, \lambda(a)=\chi_{a \cap \mathbf{Q}}(\mathbf{Q}\right.\right.$ denotes the field of rational numbers), $\nu(a):=\chi_{a \backslash \mathbf{Q}}$. Then $\mu=\lambda+\nu$ is a decomposition with the desired properties.

A further consequence of (3.3) is

(3.5) Corollary. If $\mu$ is s-bounded, $(G, \mathbf{W})$ complete and $\mathbf{U} \in$ $V(\mathbf{W}(\mu))$, then there exists a content $\nu: R \rightarrow G$ with $\mathbf{W}(\nu)=\mathbf{U}$. Moreover, if $G=[0, \infty[$, then $\nu$ can be chosen with $\nu \leq \mu$.

Proof. Choose $\lambda$ and $\nu$ as in (3.3). Then $\mathbf{W}(\mu)=\mathbf{W}(\lambda) \vee \mathbf{W}(\nu)=$ $\mathbf{W}(\lambda) \vee \mathbf{U}$. The uniqueness statement in (2.6)(3) yields $\mathbf{W}(\nu)=\mathbf{U}$. If a relation $\leq$ on $G$ is defined with $z \leq y+z(y, z \in G)$, then for $a \in R$ holds $\nu(a) \leq \lambda(a)+\nu(a)=\mu(a)$, i.e. $\nu \leq \mu$.

Special decomposition theorems can be obtained by (3.3), if $\mathbf{U}$ is the supremum of a complete ideal $\mathfrak{A}$ in $\mathfrak{M}_{s}(R)$ (or in $\mathfrak{M}(R)$ ). Then U-continuity of $\nu$ just means that $\mathbf{W}(\nu) \in \mathfrak{A}$. Transformations of $\mathbf{U}$-singularity of $\lambda$ are given in (3.7).

(3.6) Special decomposition theorems.

In (a), (b), (c) let $R_{0}$ be a Boolean ring (e.g. the power set of some set) and $R$ a subring of $R_{0}$; inf and sup are always built in $R_{0}$.

(a) Let $A$ and $B$ be subsets of $R_{0}$ with $A \vee A \subset A$ and $B \wedge B \subset B$. $\mathbf{V} \in \mathfrak{M}(R)$ is called $(A, B)$-regular, if for every $x \in R$ and $V \in \mathbf{V}$ there are elements $a \in A$ and $b \in B$ with $a \leq x \leq b$ and $\{z \in R: z \leq b \backslash a\} \subset V$. $\mu$ is called $(A, B)$-regular (with respect to $\mathbf{W})$, if $\mathbf{W}(\mu)$ is $(A, B)$-regular. Evidently $\mathfrak{A}:=\left\{\mathbf{V} \in \mathfrak{M}_{s}(R): \mathbf{V}\right.$ is $(A, B)$-regular $\}$ is a complete ideal in $\mathfrak{M}_{s}(R)$. With $\mathbf{U}:=\sup \mathfrak{A}(3.3)$ yields a decomposition $\mu=\lambda+\nu$ into a ( $A, B)$-regular content $\nu$ and a " $(A, B)$-antiregular" content $\lambda$. A much more special decomposition (of Borel measures on locally compact spaces with values in normed spaces) was obtained by Ohba [16, Theorem 3].

(b) For an infinite cardinal number $\kappa \mathfrak{A}:=\{\mathbf{V} \in \mathfrak{M}(R)$ : every decreasingly directed subset $M$ of $R$ with $|M| \leq \kappa$ and inf $M=0$ converges to 0 with respect to $\mathbf{V}\}$ is a complete ideal in $\mathfrak{M}(R)$. With $\mathbf{U}:=\sup \mathfrak{A}$ 
(3.3) yields a decomposition $\mu=\lambda+\nu$ into a content $\nu$, which is " $\kappa$-smooth with respect to $R_{0}$ ", and a " $\kappa$-singular" content $\lambda$. Specially for countable $\kappa$ we get a Hewitt-Yosida-type decomposition of $\mu$ into a content, which is " $\sigma$-additive with respect to $R_{0}$ ", and a "purely finitely additive" one (s. [21, Chapter 5]).

(c) Now we consider with regard to [3] a little more general situation as in (b). Let $\kappa$ be an infinite cardinal number and $A$ a subset of $R$ with $A \vee A \subset A$. Then $\mathfrak{A}:=\{\mathbf{V} \in \mathfrak{M}(R): \mathbf{V}$ is $(A, R)$-regular and every decreasingly directed subset $M$ of $A$ with $|M| \leq \kappa$ and inf $M=0$ converges to 0 with respect to $\mathbf{V}$ ) is a complete ideal in $\mathfrak{M}(R)$. (Specially for $A=R$ we have again the situation of (b)). With $U:=\sup \mathfrak{A}(3.3)$ yields a generalization of the decomposition theorem of [3, Chapter 3]. To see this we have only to observe Lemma (3.6.2).

(3.6.1) Lemma. For every neighbourhood $W_{0}$ of $\mu(0)$ there exist a neighbourhood $V_{0}$ of $\mu(0)$ with $x \in W_{0}$ whenever $x \in \mu(R), y \in V_{0}$ and $x+y \in V_{0}$.

Proof. Let $W \in \mathbf{W}$ be an entourage, $W_{0}=\{z \in G:(z, 0) \in W\}$. Choose $U \in \mathbf{W}$ with $U \circ U \subset W$ and a symmetric entourage $V \in \mathbf{W}$ with $V \subset U$ and $\left(x_{1}+y_{1}, x_{2}+y_{2}\right) \in U$ whenever $\left(x_{1}, x_{2}\right),\left(y_{1}, y_{2}\right) \in V$. Now let be $x \in \mu(R)$ and $y \in V_{0}:=\{z \in G:(z, 0) \in V\}$ with $x+y \in V_{0}$. $(0, y),(x, x) \in V$ implies $(x, x+y) \in U$. Since also $(x+y, 0) \in U$, we get $(x, 0) \in W$, i.e. $x \in W_{0}$.

(3.6.2) Lemma. Suppose $A$ is a lattice and $\mu$ is $(A, R)$-regular; then $\mathbf{W}(\mu) \in \mathfrak{A}$ iff for every decreasingly directed subset $M$ of $A$ with $|M| \leq \kappa$ and inf $M=0$ the set $(\mu(a))_{a \in M}$ converges to 0 .

Proof. One implication holds obviously since $\mu$ is $\mathbf{W}(\mu)$-continuous. Suppose now, the condition is fulfilled, but $\mathbf{W}(\mu) \notin \mathfrak{A}$. Then there is a decreasingly directed subset $M$ of $A$ with $|M| \leq \kappa$ and inf $M \stackrel{\prime}{=} 0$, further a closed neighbourhood $W_{0}$ of $\mu(0)$ with $\mu(R \wedge a) \not \subset W_{0}$ whenever $a \in M$; choose $V_{0}$ according to (3.6.1) and similarly a neighbourhood $U_{0}$ of $\mu(0)$ with $x \in V_{0}$ whenever $x \in \mu(R), y \in U_{0}$ and $x+y \in U_{0}$. We show:

(*) For every $a \in M$ there are elements $b_{a} \in A$ and $c_{a} \in M$ with $c_{a} \leq b_{a} \leq a$ and $\mu\left(b_{a}\right) \notin V_{0}$.

For the proof of (*) let be $a \in M$ and $d \in R \wedge a$ with $\mu(d) \notin W_{0}$. Since $\mu$ is $(A, R)$-regular and $W_{0}$ is closed, there is an element $b \in A$ with $b \leq d$ and $\mu(b) \notin W_{0}$. Since by our assumption both $(\mu(x))_{x \in M}$ and 
$(\mu(x \wedge b))_{x \in M}$ converge to 0 , there is an element $c_{a} \in A$ with $\mu\left(c_{a}\right) \in U_{0}$ and $\left(\mu\left(c_{a} \wedge b\right)\right) \in U_{0}$. Since $\mu\left(c_{a}\right)=\mu\left(c_{a} \backslash b\right)+\mu\left(c_{a} \wedge b\right)$. we get $\mu\left(c_{a} \backslash b\right) \in V_{0}$. Put $b_{a}:=c_{a} \vee b$. Then the relations $\mu\left(c_{a} \backslash b\right) \in V_{0}, \mu(b)$ $\notin W_{0}, \mu\left(b_{a}\right)=\mu(b)+\mu\left(c_{a} \backslash b\right)$ imply $\mu\left(b_{a}\right) \notin V_{0}$.

By $(*)\left\{b_{a}: a \in M\right\}$ is a decreasingly directed subset of $A$ with power $\leq \kappa$ and $\inf _{a \in M} b_{a}=0$, but $\left(\mu\left(b_{a}\right)\right)_{a \in M}$ doesn't converge to 0 , and so we get a contradiction.

(d) For $A \subset R \mathfrak{A}:=\left\{\mathbf{V} \in \mathfrak{M}_{s}(R): A \subset N(\mathbf{V})\right\}$ is a complete ideal in $\mathfrak{M}_{s}(R)$. If $R$ is a ring of sets and $A:=\{a \in R:|a| \leq \kappa\}$, then (3.3) yields with $\mathbf{U}:=\sup \mathfrak{A}$ a decomposition theorem, which in a more special case agrees with [17; Theorem 1, Theorem 3].

(e) If $\mathfrak{A}$ is the complete ideal $\mathfrak{A}:=\left\{\mathbf{V} \in \mathfrak{M}_{s}(R): \mathbf{V}\right.$ is atomic $\}$ (s. (2.13)(a)) and $\mathbf{U}:=\sup \mathfrak{A}$, then (3.3) yields a decomposition $\mu=\lambda+\nu$ into an atomic content $\nu$ and an atomless content $\lambda$ (compare [24, Theorem (6.8)]).

(f) It follows from (2.5) and (3.5) that $\mathfrak{A}_{0}:=\left\{\sup _{\alpha} \mathbf{W}_{0}\left(\mu_{\alpha}\right):\left(\mu_{\alpha}\right)_{\alpha \in A}\right.$ is a family of $[0,1]$-valued contents on $R\}$ is a complete ideal in $\mathfrak{M}_{s}(R)$; put $\mathbf{U}_{0}:=\sup \mathfrak{A}_{0}$. Whether it is always $\lambda=0$ and $\nu=\mu$ in a decomposition $\mu=\lambda+\nu$ obtained by (3.3) with $\mathbf{U}:=\mathbf{U}_{0}$, this is equivalent to the unsolved Maharam-problem considered in [2]. If $G$ is a locally convex space and $\mathbf{W}$ its uniformity, then by [25, Theorem (3.3)] $\mathbf{W}(\mu) \in \mathfrak{A}_{0}$.

\section{(3.7) Singularities.}

For a precise comparison of the decomposition theorems of this paper with corresponding theorems of other papers we have to clear the connection between $\mathbf{U}$-singularity of $\mu$ and notions such as $\kappa$-singularity in the sense of [3], antiregularity in the sense of [16] etc. In the following let $\mu$ be $s$-bounded, $\mathfrak{A}$ a complete ideal in $\mathfrak{M}_{s}(R), \mathbf{U}=\sup \mathfrak{A}$ and $\mathfrak{B} \subset \mathfrak{A}$ with sup $\mathfrak{B} \geq \mathbf{W}(\mu) \wedge \mathbf{U}$ (e.g. $\mathfrak{B}=\{\mathbf{V} \in \mathfrak{A}: \mathbf{V}$ possesses an at most countable 0 -neighbourhood base $\}$ or in case that $\mu$ is $\sigma$-smooth $\mathfrak{B}=\{\mathbf{V} \in \mathfrak{A}: \mathbf{V}$ possesses an at most countable 0 -neighbourhood base and is $\sigma$-smooth $\}$ ). Then the following statements are equivalent:

(1) $\mu$ is $\mathbf{U}$-singular.

(2) The trivial topology is the only topology, which belongs to $\mathfrak{A}$ and is coarser than $\mathbf{W}(\mu)$.

(3) $\mu$ is $\mathbf{V}$-singular for every $\mathbf{V} \in \mathfrak{B}$.

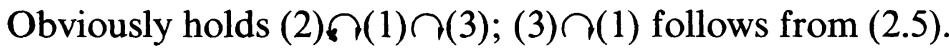

If $(G, \mathbf{W})$ is complete, a further equivalent statement is

(4) $\mu \perp \nu$ for every content $\nu: R \rightarrow G$ with $\mathbf{W}(\nu) \in \mathfrak{A}$.

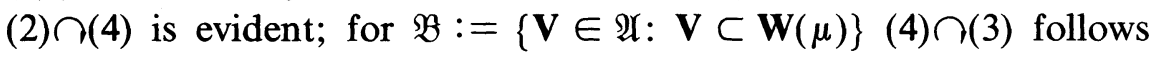
from (3.5). 
The condition (4) corresponds to the notion of $\kappa$-singularity in the sense of [3] (compare (3.6)(c), (1.4)), the condition (2) (with $\mathfrak{B}=\mathfrak{U}$ ) to the notion of purely additivity in the sense of $[21,5.1]$ (compare (3.6)(b), [21, $1.5(2)])$.

In the following we consider the case that $\mathbf{W}(\mu)$ belongs to $\mathfrak{A}_{0}, \mathfrak{A}_{0}$ defined as in (3.6)(f); this is fulfilled, if the range space of $\mu$ is a locally convex linear space. With $\mathfrak{B}:=\left\{\mathbf{W}_{0}(\nu): \nu: R \rightarrow[0, \infty[\right.$ is a content with $\left.\mathbf{W}_{0}(\nu) \in \mathfrak{U}\right\}$ we get a further equivalence to (3):

(5) $\mu \perp \nu$ for every content $\nu: R=\left[0, \infty\left[\right.\right.$ with $\mathbf{W}_{0}(\nu) \in \mathfrak{A}$.

If $\mu$ is a measure ( $=\sigma$-additive content), then for $\mathfrak{B}:=\left\{\mathbf{W}_{0}(\nu): \nu\right.$ : $R \rightarrow\left[0, \infty\left[\right.\right.$ is a measure with $\left.\mathbf{W}_{0}(\nu) \in \mathfrak{A}\right\}$ the following condition is also equivalent to (3):

(6) $\mu \perp \nu$ for every measure $\nu: R \rightarrow\left[0, \infty\left[\right.\right.$ with $\mathbf{W}_{0}(\nu) \in \mathfrak{A}$.

(6) corresponds to the notion of antiregularity in the sense of [16].

If $G$ is a locally convex space and $G^{\prime}$ its topological dual, then by [25, Theorem (3.3)] $\mathbf{W}(\mu)=\sup _{x^{\prime} \in G^{\prime}} \mathbf{W}_{0}\left(x^{\prime} \circ \mu\right)$ and so the next condition (7) is equivalent to (5) as can be seen using (2.5).

(7) $x^{\prime} \circ \mu \perp \nu$ for every $x^{\prime} \in G^{\prime}$ and every content $\nu: R \rightarrow[0, \infty[$ with $\mathbf{W}_{0}(\nu) \in \mathfrak{U}$.

If $\mu$ is a real-valued content of bounded variation $|\mu|$, then, because of (3.5), the next statement is equivalent to (2).

(8) For every content $\nu: R \rightarrow\left[0, \infty\left[\mathbf{W}_{0}(\nu) \in \mathfrak{A}\right.\right.$ and $0 \leq \nu \leq|\mu|$ imply $\nu=0$.

(7), (8) correspond to the notion of $[4$, p. 30] and [22; Theorems 4 and 5].

Brooks [1, Theorem 1] proved a Hewitt-Yosida-type decomposition $\mu=\lambda+\nu$ for a bounded content with values in a Banach space $X$, where $\mu$ is not necessary $s$-bounded with respect to the norm topology of $X$; the values of $\lambda$ and $\nu$ belong to the bidual $X^{\prime \prime}$ of $X$. Decompositions of this kind one can obtain from (3.3) considering $\mu$ as $X^{\prime \prime}$-valued content, where $X^{\prime \prime}$ is endowed with the weak star topology; with respect to this topology $\mu$ is $s$-bounded; to prove that the values of $\lambda$ and $\nu$ belong to $X^{\prime \prime}$ (and not only $X^{\prime *}$ ), observe (3.4) and that every bounded weak Cauchy net in $X$ is convergent in $X^{\prime \prime}$.

In the following we consider decompositions of $\mu$ into an infinite sum instead of decompositions into a sum of two contents.

(3.8) Proposition. Let $\mu$ be s-bounded and $(G, \mathbf{W})$ complete. We use the notation $\tilde{R}, \tilde{\mathbf{U}}_{s}, \tilde{\mu}, m$ as in (3.1). Let $\left(u_{\alpha}\right)_{\alpha \in A}$ be a disjoint family in $\tilde{R}$ with $\sup _{\alpha} u_{\alpha}=e$. (Observe that $\tilde{R}$ has $a$ unit $e$ by $(2.2)(\mathrm{b})$ ). Put 
$\mu_{\alpha}(x):=\tilde{\mu}\left(x \wedge u_{\alpha}\right)$ for $\alpha \in A$ and $x \in R$. Then we have:

(a) For $\alpha \in A \mu_{\alpha}$ is a s-bounded content, $\mu_{\alpha}(0)=0, \mu_{\alpha}(R) \subset \overline{\mu(R)}$.

(b) $\mu_{\alpha} \perp \mu_{\beta}, \mu_{\alpha}+\mu_{\beta}=\mu_{\beta}+\mu_{\alpha},\left(\mu_{\alpha}+\mu_{\beta}\right)+\mu_{\gamma}=\mu_{\alpha}+\left(\mu_{\beta}+\mu_{\gamma}\right)$ for different $\alpha, \beta, \gamma \in A$.

(c) $\mathbf{W}\left(\mu_{\alpha}\right)=\pi\left(m \wedge u_{\alpha}\right)$ for $\alpha \in A$. W( $(\mu)=\sup _{\alpha \in A} \mathbf{W}\left(\mu_{\alpha}\right)$.

(d) $\mu=\Sigma_{\alpha \in A} \mu_{\alpha} ; \Sigma_{\alpha \in A} \mu_{\alpha}(x)$ converges to $\mu(x)$ uniformly in $x \in R$, i.e. for every $W \in \mathbf{W}$ there exists a finite set $A_{0} \subset A$, such that $(\mu(x)$, $\left.\Sigma_{\alpha \in B} \mu_{\alpha}(x)\right) \in W$ whenever $B$ is a finite set with $A_{0} \subset B \subset A$ and $x \in R$.

Proof. From (3.1) follows (a) and $\mathbf{W}\left(\mu_{\alpha}\right)=\pi\left(m \wedge u_{\alpha}\right)(\alpha \in A)$. The equalities $m=\sup _{\alpha}\left(m \wedge u_{\alpha}\right)$ and $\left(m \wedge u_{\beta}\right) \wedge\left(m \wedge u_{\gamma}\right)=0$ (for different $\beta, \gamma \in A)$ just mean that $\mathbf{W}(\mu)=\sup _{\alpha} \mathbf{W}\left(\mu_{\alpha}\right)$ and $\mathbf{W}\left(\mu_{\beta}\right), \mathbf{W}\left(\mu_{\gamma}\right)$ are singular (s. $(2.2)(\mathrm{c}))$. The associative and commutative statements in (b) hold obviously.

(d) Let be $W \in \mathbf{W}$. Since $\tilde{\mu}$ is uniformly continuous, there is a normal set $U \in \tilde{\mathbf{U}}_{s}$ such that $(\tilde{\mu}(x), \tilde{\mu}(y)) \in W$ whenever $x, y \in \tilde{R}$ and $x \triangle y \in U$. Because of the $\tau$-smoothness of $\tilde{\mathbf{U}}_{s}$ (s. (2.2)(b)) there is a finite subset $A_{0}$ of $A$ with $e \backslash \sup _{\alpha \in A_{0}} u_{\alpha} \in U$. Now, for $x \in R$ and $A_{0} \subset B \subset A$ with $|B|<$ $\infty$, we have $x \Delta\left(x \wedge \sup _{\alpha \in B} u_{\alpha}\right) \leq e \backslash \sup _{\alpha \in A_{0}} u_{\alpha}$, hence $x \Delta(x \wedge$ $\left.\sup _{\alpha \in B} u_{\alpha}\right) \in U$ and $\left(\mu(x), \Sigma_{\alpha \in B} \mu_{\alpha}(x)\right)=\left(\tilde{\mu}(x), \tilde{\mu}\left(x \wedge \sup _{\alpha \in B} u_{\alpha}\right)\right) \in$ $W$.

The following decomposition in case that $\mu$ is a measure on a $\sigma$-algebra with values in a metrizable group goes back to Herer [10].

(3.9) COROLlaRY. If $\mu$ is $s$-bounded and $(G, \mathbf{W})$ complete, then there is a family of s-bounded, pairwise singular contents $\mu_{\alpha}: R \rightarrow G(\alpha \in A)$ with the following properties:

(1) $\mu_{\alpha}+\mu_{\beta}=\mu_{\beta}+\mu_{\alpha},\left(\mu_{\alpha}+\mu_{\beta}\right)+\mu_{\gamma}=\mu_{\alpha}+\left(\mu_{\beta}+\mu_{\gamma}\right)$ for different $\alpha, \beta, \gamma \in A . \mu=\Sigma_{\alpha \in A} \mu_{\alpha}$, where $\Sigma_{\alpha} \mu_{\alpha}(x)$ converges to $\mu(x)$ uniformly in $x \in R$. W $(\mu)=\sup _{\alpha \in A} \mathbf{W}\left(\mu_{\alpha}\right)$.

(2) $N\left(\mu_{\alpha}\right)=\left\{x \in R: \mu_{\alpha}(x)=0\right\}$ for all $\alpha \in A$.

Proof. We use the notations of (3.8); put $N_{0}:=\tilde{\mu}^{-1}(0)$ and $N:=\{x$ $\left.\in R:(\tilde{R} \wedge u) \cap N_{0} \subset N(\tilde{\mu})\right\}$. First we show that $\sup N=e$.

If $s:=\sup N \neq e$, then $e \backslash s \notin N(\tilde{\mu}) \subset N$ and so $\tilde{\mu}(a) \neq 0$ for some $a \in \tilde{R} \backslash s$. Let $D$ be a maximal disjoint subset of $N_{0} \cap(\tilde{R} \wedge a)$. Since $N_{0}$ is closed and $x \vee y \in N_{0}$ for disjoint elements $x, y \in N_{0}$, we have $t:=\sup D \in N_{0}$ (s. (2.2)(b)). The maximality of $D$ implies $a \backslash t \in N$, hence $a \backslash t \leq s$ and therefore $t=a$ because of $t \leq a \in \tilde{R} \backslash s$. Now we get $a=t \in N_{0}, \tilde{\mu}(a)=0$, a contradiction. Since $\sup N=e$ and $N$ is normal, 
there is by $[19,20.2]$ a disjoint family $\left(u_{\alpha}\right)_{\alpha \in A}$ in $N$ with $\sup _{\alpha} u_{\alpha}=e$. Now define $\mu_{\alpha}$ as in (3.8), then the statements (1) and (2) are satisfied.

(3.10) THEOREM. Let $\mu$ be $s$-bounded, $(G, \mathbf{W})$ complete and $\mathbf{U}_{\alpha} \in \mathfrak{M}(R)$ for $\alpha \in A$. Then there are $G$-valued, s-bounded contents $\lambda$ and $\mu_{\alpha}(\alpha \in A)$ with the following properties:

(1) $\mu_{\alpha}$ is $\mathbf{U}_{\alpha}$-continuous and $\lambda \mathbf{U}_{\alpha}$-singular for $\alpha \in A$.

(2) $\mu_{\alpha}+\mu_{\beta}=\mu_{\beta}+\mu_{\alpha},\left(\mu_{\alpha}+\mu_{\beta}\right)+\mu_{\gamma}=\mu_{\alpha}+\left(\mu_{\beta}+\mu_{\gamma}\right)$ for different $\alpha, \beta, \gamma \in A$.

(3) $\mu=\lambda+\Sigma_{\alpha \in A} \mu_{\alpha}$, where $\Sigma_{\alpha} \mu_{\alpha}(x)$ converges uniformly in $x \in R$. $\lambda(0)=\mu_{\alpha}(0)=0$ for $\alpha \in A$.

(4) $\mathbf{W}(\mu)=\mathbf{W}(\lambda) \vee \sup _{\alpha \in A} \mathbf{W}\left(\mu_{\alpha}\right) . \lambda(R), \mu_{\alpha}(R) \subset \overline{\mu(R)} ; \lambda+\mu_{\alpha}=$ $\mu_{\alpha}+\lambda ; \mu_{\alpha} \perp \lambda(\alpha \in A) . \mu_{\alpha} \perp \mu_{\beta}$ for different $\alpha, \beta \in A$.

$\lambda$ and $\Sigma_{\alpha} \mu_{\alpha}$ are uniquely determined by (1), (2), (3). If the topologies $\mathbf{U}_{\alpha}$, $\alpha \in A$, are pairwise singular, then the family $\left(\mu_{\alpha}\right)_{\alpha \in A}$ is also uniquely determined by (1), (2), (3).

Proof. As in the proof of (3.3) we may assume that $\mathbf{U}_{\alpha}$ is $s$-bounded for all $\alpha \in A$. Put $\mathbf{U}:=\sup _{\alpha} \mathbf{U}_{\alpha}$ and choose $\mathbf{V} \in \mathfrak{M}_{s}(R)$ such that $\mathbf{V} \vee \mathbf{U}$ $=\mathbf{U}_{s}$ and $\mathbf{V} \wedge \mathbf{U}$ is trivial (s. (2.5)). That a content $\lambda$ is $\mathbf{U}_{\alpha}$-singular for all $\alpha \in A$, just mean that $\lambda$ is $\mathbf{U}$-singular or, equivalently to this, that $\lambda$ is V-continuous.

To prove the existence statement we may therefore admit $\mathbf{V}$ to the family $\left(\mathbf{U}_{\alpha}\right)_{\alpha \in A}$ such that $\mathbf{U}_{s}=\sup _{\alpha} \mathbf{U}_{\alpha}$; further, in view of (2.9)(a) we may assume that the topologies $\mathrm{U}_{\alpha}, \alpha \in A$, are pairwise singular. By the notations of (3.8) $u_{\alpha}:=\pi^{-1}\left(\mathbf{U}_{\alpha}\right)$ are pairwise disjoint and $e=\sup _{\alpha} u_{\alpha}$. Now, choose $\mu_{\alpha}$ as in (3.8); then the statements (1), (2), (3), (4) (with $\lambda=0$ ) evidently hold by (3.8). (Observe that $m \wedge u_{\alpha} \leq u_{\alpha}$ just mean $\mathbf{W}\left(\mu_{\alpha}\right) \subset \mathbf{U}_{\alpha}$.) To prove the uniqueness statement let $\lambda, \mu_{\alpha}$ be given with (1), (2), (3). Then $\nu:=\sum_{\alpha \in A} \mu_{\alpha}$ is a content. $\nu$ is U-continuous, especially $s$-bounded: Let be $W \in \mathbf{W}$ and $W_{0}:=\{z \in G:(z, 0) \in W\}$. Choose $V \in \mathbf{W}$ with $V \circ V \subset W$, a finite subset $B$ of $A$ with $\left(\nu(x), \Sigma_{\alpha \in B} \mu_{\alpha}(x)\right) \in$ $V$ for $x \in R$, further $U \in \mathbf{U}$ such that $U \subset\left\{a \in R:\left(\Sigma_{\alpha \in B} \mu_{\alpha}\right)(R \wedge a) \subset\right.$ $\left.V_{0}\right\}$ where $V_{0}:=\{z \in G:(z, 0) \in V\}$; then $U \subset\{a \in R: \nu(R \wedge a) \subset$ $\left.W_{0}\right\}$. Now the uniqueness of $\lambda$ and $\Sigma_{\alpha \in A} \mu_{\alpha}$ follows from the uniqueness statement in (3.3). If the topologies $\mathbf{U}_{\alpha}, \alpha \in A$, are pairwise singular, put $u_{\alpha}:=\pi^{-1}\left(\mathbf{U}_{\alpha}\right)$ and $v:=\pi^{-1}(\mathbf{V})$; let be $\tilde{\lambda}, \tilde{\mu}_{\alpha}$ the continuous extension of $\lambda$, $\mu_{\alpha}$ on $\left(\tilde{R}, \tilde{\mathbf{U}}_{s}\right)$, respectively. Since $\Sigma_{\alpha} \mu_{\alpha}(x)$ converges uniformly in $x \in R, \Sigma_{\alpha} \tilde{\mu}_{\alpha}(x)$ converges uniformly in $x \in \tilde{R}$ and $\tilde{\mu}=\tilde{\lambda}+\Sigma_{\alpha \in A} \tilde{\mu}_{\alpha}$. As in the proof of (3.3) one can show that $\lambda(x)=\tilde{\mu}(x \wedge v), \mu_{\alpha}(x)=$ $\tilde{\mu}\left(x \wedge u_{\alpha}\right)$ for $x \in R$ and $\alpha \in A$. 
For $|A|=1$ Theorem (3.10) agrees with Theorem (3.3). A statement analogic to (3.4) is also true for decompositions according to (3.10). If $\mathbf{W}(\mu)$ possesses a 0 -neighbourhood base of power $\leq \kappa$ (this is fulfilled, if $\mathbf{W}$ has a base of power $\leq \kappa)$, then in the decompositions of (3.8), (3.9), (3.10) the system of the contents $\mu_{\alpha}$ different from 0 has a power $\leq \kappa$ (s. $(2.10)(1) \cap(3))$.

Special decompositions of contents in an infinite sum were given by Artemenko (s. [15, 1 on p. 197]), Phillips [17, Theorem 2], Rickart [18, Theorem 3.8], by Hammer and Sobczyk [9, Theorem 4.1] and in [24, Theorem (6.9)]; all these theorems can be obtained by (3.10) (or (3.9)).

We only note one special case of (3.10) (compare [9, 4.1], [24; (6.8), (6.9)]):

(3.11) COROLlaRY. If $\mu$ is s-bounded and $(G, \mathbf{W})$ complete, then there are $G$-valued, s-bounded contents $\lambda, \mu_{\alpha}(\alpha \in A)$ with the following properties:

(1) The conditions (2), (3), (4) of (3.10) are fulfilled; especially $\mu=\lambda+$ $\Sigma_{\alpha \in A} \mu_{\alpha}$.

(2) $\lambda$ is atomless. For all $\alpha \in A \mathbf{W}\left(\mu_{\alpha}\right)$ is an ultrafilter topology; especially $\left|\mu_{\alpha}(R)\right|=2$.

Further $\lambda$ can be decomposed in the form $\lambda=\Sigma_{\beta \in B} \lambda_{\beta}$, where $\left(\lambda_{\beta}\right)_{\beta \in B}$ is a family of G-valued, s-bounded, pairwise singular contents on $R$ with the following properties: $\lambda_{\alpha}+\lambda_{\beta}=\lambda_{\beta}+\lambda_{\alpha},\left(\lambda_{\alpha}+\lambda_{\beta}\right)+\lambda_{\gamma}=\lambda_{\alpha}+$ $\left(\lambda_{\beta}+\lambda_{\gamma}\right)$ for different $\alpha, \beta, \gamma \in B ; \sum_{\beta \in B} \lambda_{\beta}(x)$ converges uniformly in $x \in R ; \mathbf{W}(\lambda)=\sup _{\beta \in B} \mathbf{W}\left(\lambda_{\beta}\right) ;$ for $\beta \in B \mathbf{W}\left(\lambda_{\beta}\right)$ possesses a countable 0-neighbourhood base, consequently $\lambda_{\beta}$ satisfies CCC (i.e. $R / N\left(\lambda_{\beta}\right)$ satisfies the $\sigma$-chain condition).

(3.11) follows immediately from (3.10), (2.14), (2.10). Because of (3.9) it is even possible to choose $\left(\lambda_{\beta}\right)_{\beta \in B}$ in (3.11) in such a way that $N\left(\lambda_{\beta}\right)=\lambda_{\beta}^{-1}(\{0\})$ for all $\beta \in B$.

Using the terminology of [14] every content $\mu: \mathbf{P}(N) \rightarrow[0, \infty[$ on the power set of $\mathbf{N}$ has by (3.11) a decomposition into a "full-valued" content $\lambda$ and a "generalized ultrafilter content" $\Sigma_{\alpha \in A} \mu_{\alpha}$.

In (3.3), (3.6), (3.10), (3.11) we have given decompositions of $\mu$ with respect to $\mathbf{W}$. If we consider except of the uniformity $\mathbf{W}$ (which is complete in these theorems) another separated uniformly $\mathbf{W}^{\prime}$ coarser than $\mathbf{W}$, such that + is also uniformly continuous with respect to $\mathbf{W}^{\prime}$, then the decompositions with respect to $\mathbf{W}$ established in these theorems are also decompositions with respect to $\mathbf{W}^{\prime}$. Indeed, if $\mu$ is $s$-bounded with respect to $\mathbf{W}$, $(G, \mathbf{W})$ complete and $\mathbf{W}^{\prime} \subset \mathbf{W}$, then by $[\mathbf{2 5},(3.2)] \mathbf{W}(\mu)=\mathbf{W}^{\prime}(\mu)$; similar statements hold for $\lambda, \nu, \mu_{\alpha}, \lambda_{\beta}$. 
Now we deal with the question, whether a Lebesgue decomposition is also valid for not necessary $s$-bounded, e.g. locally $s$-bounded contents. As we will see in (3.13), the answer is no.

(3.12) Proposition. If $\lambda$ and $\nu$ are $G$-valued contents on $R$ with $\lambda \perp \nu$ and $\mu=\lambda+\nu$, then $\mathbf{W}(\mu)=\mathbf{W}(\lambda) \vee \mathbf{W}(\nu)$.

Proof. Obviously we have $\mathbf{W}(\mu) \subset \mathbf{W}(\lambda) \vee \mathbf{W}(\nu)$ (s. [24, (6.1)(b)]). If $\mathbf{W}(\lambda) \not \subset \mathbf{W}(\mu)$, then there is a neighbourhood $U_{0}$ of $\mu(0)$ such that for every neighbourhood $V_{0}$ of $\mu(0) \quad\left\{a \in R: \mu(R \wedge a) \subset V_{0}\right\} \not \subset\{a \in R$ : $\left.\lambda(R \wedge a) \subset U_{0}\right\}$. Choose a neighbourhood $W_{0}$ of $\mu(0)$ with $W_{0}+W_{0} \subset U_{0}$ and then $V_{0}$ according to (3.6.1). For some $a \in R$ it is $\mu(R \wedge a) \subset V_{0}$, but $\lambda(a) \notin U_{0}$. Since $\lambda \perp \nu$ there are disjoint elements $b, c \in R$ such that $a=b \vee c, \lambda(b) \in W_{0}$ and $\nu(c) \in V_{0}$. Then $\mu(c), \nu(c) \in V_{0}$ and $\mu(c)=$ $\lambda(c)+\nu(c)$ imply $\lambda(c) \in W_{0}$ (s. (3.6.1)), hence $\lambda(a)=\lambda(b)+\lambda(c) \in W_{0}$ $+W_{0} \subset U_{0}$ and we get a contradiction. This proves $\mathbf{W}(\lambda) \subset \mathbf{W}(\mu)$; similarly $\mathbf{W}(\nu) \subset \mathbf{W}(\mu)$.

(3.13) COROLlaRY. If $(G, \mathbf{W})$ is complete, then the following statements are equivalent:

(1) $\mu$ is s-bounded.

(2) For every $\mathbf{U} \in \mathfrak{M}(R)$ there is a unique decomposition $\mu=\lambda+\nu$ of $\mu$ into a $\mathrm{U}$-singular content $\lambda: R \rightarrow G$ and $a \mathrm{U}$-continuous content $\nu: R \rightarrow G$ with $\lambda(0)=\mu(0)=0$.

(3) For every $\mathbf{U} \in V(\mathbf{W}(\mu))$ there is a decomposition $\mu=\lambda+\nu$ of $\mu$ into a U-singular content $\lambda: R \rightarrow G$ and a U-continuous content $\nu: R \rightarrow G$.

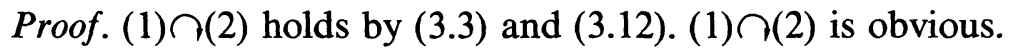

(3) (1): Let be $\mathbf{U} \in V(\mathbf{W}(\mu))$ and $\lambda, \mu$ chosen according to (3). Then $\lambda \perp \nu$, hence by (3.12) $\mathbf{W}(\mu)=\mathbf{W}(\lambda) \vee \mathbf{W}(\nu)=\mathbf{W}(\lambda) \vee \mathbf{U}$ and $\mathbf{W}(\lambda), \mathbf{U}$ are singular. This proves that $V(\mathbf{W}(\mu))$ is a complemented lattice; hence $\mathbf{W}(\mu)$ is $s$-bounded by (2.6).

Finally some words to the work of Graves ([8a], [8b]), starting point of which is the observation that for a given complete, Hausdorff, locally convex space $W$ and an algebra $R$ of sets there are natural isomorphisms $\phi \leftrightarrow \tilde{\phi} \leftrightarrow \hat{\phi}$ between the space $\operatorname{sca}(R, W)$ of all $s$-bounded, $W$-valued measures on $R$, the space $L((S(R), \tau), W)$ of all continuous linear maps from $(S(R), \tau)$ into $W$ and the analogically defined space $L(\widehat{S(R)}, W)$; here the following notations are used: $S(R):=\left\{\sum_{i=1}^{n} \alpha_{i} \chi_{A}: n \in \mathbf{N}, \alpha_{i}\right.$ real, $\left.A_{i} \in R\right\}, \tilde{\phi}(f):=\int f d \phi$ for $\phi \in \operatorname{sca}(R, W)$ and $f \in S(R) ; \tau$ denotes the coarsest locally convex topology on $S(R)$ such that $\tilde{\phi}$ is continuous for 
every $s$-bounded measure $\phi: R \rightarrow X$ into any locally convex $X, \widehat{S(R)}$ the completion of $(S(R), \tau)$ and $\hat{\phi}$ the continuous extension of $\tilde{\phi}$ on $\widehat{S(R)}$. The aim of [8] is a topologically linearizing of the study of $s$-bounded vector measures by using the isomorphism $\phi \leftrightarrow \hat{\phi}$. Now to the Lebesgue decomposition: In the former version [8a] of Graves' theory measures have decomposed by ideals, following Traynor [20]. In the sketch [8b] of Graves' refined theory a Lebesgue decomposition for locally convexvalued, $s$-bounded measures is obtained which is (for such contents) essentially equivalent to (3.3); the proof rests on still unpublished papers of Brook and Graves, cited in [8b]. The connection to our paper is the following: Identifying the sets from $R$ with the corresponding characteristic functions it is $\tau \mid R=\sup \left\{\mathbf{W}_{0}(\nu): \nu: R \rightarrow[0, \infty[\right.$ is a measure $\}$ and the set $\mathcal{P}$ of all idempotents in $\widehat{S(R)}$ is the completion of $(R, \tau \mid R)$.

\section{REFERENCES}

[1] J. K. Brooks, Decomposition theorems for vector measures, Proc. Amer. Math. Soc., 21 (1969), 27-29.

[2] J. P. R. Christensen, Some results with relation to the control measure problem, In Measure Theory (Oberwolfach 1979), Lecture Notes in Math., vol. 794, ed. A. Dold, B. Eckmann, D. Kölzow.

[3] J. A. Crenshaw and R. B. Kirk, Group valued, $\alpha$-additive set functions, Studia Math., 65 (1979), 89-101.

[4] J. Diestel and J. J. Uhl, Jr., Vector measures, Amer. Math. Soc., Math. surveys, no. 15 (1977).

[5] L. Drewnowski, Decomposition of set functions, Studia Math., 48 (1973), 23-48.

[6] _ On control submeasures and measures, Studia Math., 50 (1974), 203-224.

[7] P. Erdös and A. Tarski, On families of mutually exclusive sets, Ann. Math., 44 (1943), 315-329.

[8a] W. Graves, On the theory of vector measures, Amer. Math. Soc., Memoirs 195 (1977).

[8b] _ Vector-valued measures, In Measure Theory and Its Applications, Proceedings of the 1980 Conference, Northern Illinois University.

[9] P. C. Hammer and A. Sobczyk, A decomposition of additive set functions, Duke Math. J., 11 (1944), 839-846.

[10] W. Herer, Hahn decomposition of measures with values in a topological group, Bull. Acad. Polon. Sci. Ser. Sci. Math. Astr. Phys., 20 (1972), 203-205.

[11] S. S. Khurana, Submeasures and decomposition of measures, J. Math. Analysis Appl., 70 (1979), 111-113.

[12] I. Kluvanek and G. Knowles, Vector Measures and Control Systems, North Holland, Amsterdam (1975).

[13] Z. Lipecki, Decomposition theorems for Boolean rings, with applications to semigroupvalued measures, Ann. Soc. Math. Polon. Ser. I: Comm. Math., 20 (1978), 397-403.

[14] D. Maharam, Finitely additive measures on the integers, Sankhya, Ser. A, 38 (1976), 44-56.

[15] R. D. Mauldin, A representation theorem for the second dual of $C[0,1]$. Studia Math., 46 (1973), 197-200.

[16] S. Ohba, The decomposition theorems for vector measures, Yokohama Math. J., 19 (1) (1971), 23-28. 
[17] R. S. Phillips, A decomposition of additive set functions, Bull. Amer. Math. Soc., 46, 1 (1940), 274-277.

[18] C. E. Rickart, Decomposition of additive set functions, Duke Math. J., 10 (1943), 653-665.

[19] R. Sikorski, Boolean Algebras, Springer-Verlag, Berlin 1964.

[20] T. Traynor, Decomposition of group-valued additive set functions, Ann. Inst. Fourier (Grenoble), 22 (1972), fasc. 3, 131-140.

[21] $\_$The Lebesgue decomposition for group-valued set functions, Trans. Amer. Math. Soc., 220 (1976), 307-319.

[22] J. J. Uhl, Jr., Extensions and decompositions of vector measures, J. London Math. Soc., (2), 3 (1971), 672-676.

[23] H. Weber, Zu einem Problem von Kowalsky, Abh. Braunschweig. Wiss. Gesellschaft, 24 (1978), 127-134.

[24] _ Die atomare Struktur topologischer Boolescher Ringe und s-beschränkter Inhalte, Studia Math., 74 (1982), 57-81.

[25] _ Vergleich monotoner Ringtopologien und absolute Statigkeit von Inhalten, Comment. Math. Univ. St. Pauli, 31 (1982), 49-60.

Received June 11, 1982.

UNIVERSITÄT KONSTANZ

POSTFACH 5560

D-7750 KONSTANZ 1 



\title{
PACIFIC JOURNAL OF MATHEMATICS EDITORS
}

\author{
DONALD BABBITT (Managing Editor) \\ University of California \\ Los Angeles, CA 90024 \\ Hugo Rossi \\ University of Utah \\ Salt Lake City, UT 84112 \\ C. C. MOORE and Arthur Ogus \\ University of California \\ Berkeley, CA 94720
}

\author{
J. Dugundir \\ Department of Mathematics \\ University of Southern California \\ Los Angeles, CA 90089-1113
}

R. FINN and H. SAMELSON

Stanford University

Stanford, CA 94305

ASSOCIATE EDITORS
R. ARENS
E. F. BECKENBACH
B. H. NeumanN
F. WOLF
K. YosHIDA (1906-1982)

\section{SUPPORTING INSTITUTIONS}

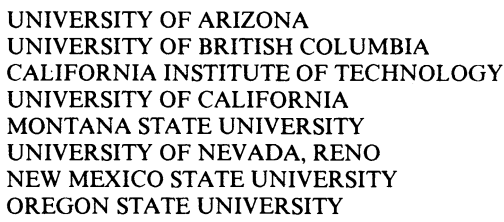

\author{
UNIVERSITY OF OREGON \\ UNIVERSITY OF SOUTHERN CALIFORNIA \\ STANFORD UNIVERSITY \\ UNIVERSITY OF HAWAII \\ UNIVERSITY OF TOKYO \\ UNIVERSITY OF UTAH \\ WASHINGTON STATE UNIVERSITY \\ UNIVERSITY OF WASHINGTON
}

The Supporting Institutions listed above contribute to the cost of publication of this Journal, but they are not owners or publishers and have no responsibility for its content or policies.

Mathematical papers intended for publication in the Pacific Journal of Mathematics should be in typed form or offset-reproduced (not dittoed), double spaced with large margins. Please do not use built up fractions in the text of the manuscript. However, you may use them in the displayed equations. Underline Greek letters in red, German in green, and script in blue. The first paragraph must be capable of being used separately as a synopsis of the entire paper. In particular it should contain no bibliographic references. Please propose a heading for the odd numbered pages of less than 35 characters. Manuscripts, in triplicate, may be sent to any one of the editors. Please classify according to the scheme of Math. Reviews, Index to Vol. 39. Supply name and address of author to whom proofs should be sent. All other communications should be addressed to the managing editor, or Elaine Barth, University of California, Los Angeles, California 90024.

There are page-charges associated with articles appearing in the Pacific Journal of Mathematics. These charges are expected to be paid by the author's University, Government Agency or Company. If the author or authors do not have access to such Institutional support these charges are waived. Single authors will receive 50 free reprints; joint authors will receive a total of 100 free reprints. Additional copies may be obtained at cost in multiples of 50 .

The Pacific Journal of Mathematics is issued monthly as of January 1966. Regular subscription rate: \$132.00 a year (6 Vol., 12 issues). Special rate: $\$ 66.00$ a year to individual members of supporting institutions.

Subscriptions, orders for numbers issued in the last three calendar years, and changes of address should be sent to Pacific Journal of Mathematics, P.O. Box 969, Carmel Valley, CA 93924, U.S.A. Old back numbers obtainable from Kraus Periodicals Co., Route 100, Millwood, NY 10546.

The Pacific Journal of Mathematics ISSN 0030-8730 is published monthly by the Pacific Journal of Mathematics at P.O. Box 969, Carmel Valley, CA 93924. Application to mail at Second-class postage rates is pending at Carmel Valley, California, and additional mailing offices. Postmaster: Send address changes to Pacific Journal of Mathematics, P. O. Box 969, Carmel Valley, CA 93924.

PUBLISHED BY PACIFIC JOURNAL OF MATHEMATICS, A NON-PROFIT CORPORATION

Copyright $(1984$ by Pacific Journal of Mathematics 


\section{Pacific Journal of Mathematics}

Vol. 110, No. $2 \quad$ October, 1984

Robert A. Bekes, The range of convolution operators $\ldots \ldots \ldots \ldots \ldots 257$

Dennis K. Burke and Sheldon Davis, Subsets of ${ }^{\omega} \omega$ and generalized metric

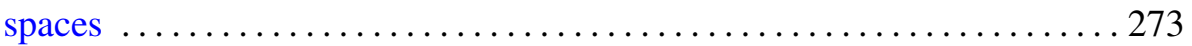

Giovanni Emmanuele, A remark on a paper: "Common fixed points of nonexpansive mappings by iteration" $\ldots \ldots \ldots \ldots \ldots \ldots \ldots \ldots \ldots 283$

I. Erdélyi and Sheng-Wang Wang, On strongly decomposable operators . . . 287

Gerhard Gierz, Injective Banach lattices with strong order units . . . . . . . 297

Maurizio Letizia, Quotients by complex conjugation of nonsingular quadrics and cubics in $\mathbf{P}_{\mathbf{C}}^{3}$ defined over $\mathbf{R} \ldots \ldots \ldots \ldots \ldots \ldots \ldots \ldots \ldots \ldots$

P. H. Maserick and Franciszek Hugon Szafraniec, Equivalent definitions

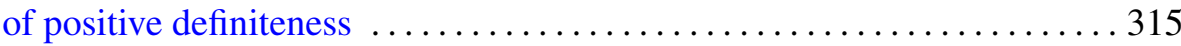

Costel Peligrad and S. Rubinstein, Maximal subalgebras of $C^{*}$-crossed

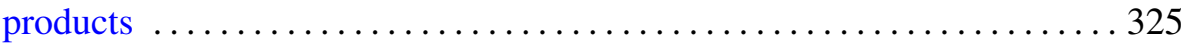

Derek W. Robinson and Sadayuki Yamamuro, Hereditary cones, order

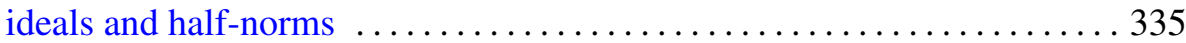

Derek W. Robinson and Sadayuki Yamamuro, The Jordan decomposition

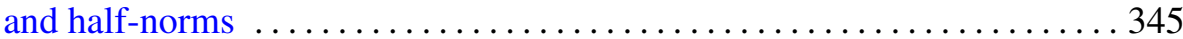

Richard Rochberg, Interpolation of Banach spaces and negatively curved

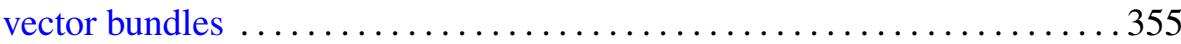

Dale Rolfsen, Rational surgery calculus: extension of Kirby's theorem 377

Walter Iaan Seaman, Helicoids of constant mean curvature and their Gauss maps

Diana Shelstad, Endoscopic groups and base change $\mathbf{C} / \mathbf{R}$

Jerrold Norman Siegel and Frank Williams, Numerical invariants of homotopies into spheres

Alladi Sitaram, Some remarks on measures on noncompact semisimple Lie groups

Teruhiko Soma, Atoroidal, irreducible 3-manifolds and 3-fold branched coverings of $S^{3}$

Jan de Vries, On the $G$-compactification of products

Hans Weber, Topological Boolean rings. Decomposition of finitely additive set functions 